\title{
Arbitrage in markets with bid-ask spreads
}

\section{The fundamental theorem of asset pricing in finite discrete time markets with bid-ask spreads and a money account}

\author{
Przemysław Rola ${ }^{1}$
}

Received: 11 July 2014 / Accepted: 3 August 2015 / Published online: 18 August 2015

(C) The Author(s) 2015. This article is published with open access at Springerlink.com

\begin{abstract}
In this paper a finite discrete time market model with bid-ask spreads and a money account is considered in the setting of an arbitrary state space. The notions of an equivalent bid-ask martingale measure (EBAMM) and of supermartingale as well as submartingale consistent price systems are introduced. The fundamental theorem of asset pricing is proved using EBAMM as an equivalent condition for no-arbitrage. The Cox-Ross-Rubinstein model with bid-ask spreads is presented as an application of our results.
\end{abstract}

Keywords Arbitrage - Bid-ask spreads - Consistent price system · Bid-ask martingale measure

\section{JEL Classification G10}

\section{Introduction}

The fundamental theorem of asset pricing, often called the Dalang-Morton-Willinger theorem, states that for the standard discrete-time finite horizon model of security market, there is no arbitrage if and only if the price process is a martingale with respect to an equivalent probability measure. A much more challenging problem in mathematical finance turned out to be finding conditions equivalent to the absence of arbitrage in markets with friction. Nowadays there are many important papers on this subject. We briefly recall some of them, devoted to multi-asset discrete-time models

\footnotetext{
$凶$ Przemysław Rola przemyslaw.rola@outlook.com

1 Faculty of Mathematics and Computer Science, Jagiellonian University, ul. Łojasiewicza 6, 30-348 Kraków, Poland
} 
with friction. For more detailed references we refer the reader e.g. to Kabanov and Safarian (2009).

Kabanov et al. (2002) gave equivalent conditions for the absence of so-called weak arbitrage opportunities (i.e. strict no-arbitrage) under the assumption of efficient friction. A general version of this theorem was proved by Kabanov and Stricker (2001b) but in the model with finite state space $\Omega$. Soon after, in his famous paper Schachermayer (2004) gave equivalent conditions for so-called robust no-arbitrage. The general theorem states that robust no-arbitrage is equivalent to the existence of a strictly consistent price system (CPS). Moreover, robust no-arbitrage cannot be replaced by strict no-arbitrage due to a counter-example presented by Schachermayer (2004). Further in this direction, a very interesting and surprising theorem was proved by Grigoriev (2005) who generalised the result of Kabanov and Stricker (2001b) to arbitrary $\Omega$ in the special case of two assets.

One of the corollaries of that theorem states that in a market with bid and ask scalar processes $S^{b}, S^{a}$ and with a money account, the absence of arbitrage is equivalent to the existence of a process $\tilde{S}$ which is a martingale under an equivalent probability measure and satisfies $S^{b} \leq \tilde{S} \leq S^{a}$. In our terminology (similar to that of Jouini and Kallal 1995) this means that no arbitrage in the case of one risky asset and a money account is equivalent to the existence of a CPS. The question whether one can extend this result to markets with a money account and arbitrary $d$ risky assets remains open. However, Grigoriev (2005) suggested that the answer to this question seems to be negative. One of the purposes of our paper is to analyse the general model of market with bid-ask prices and a money account in order to research this issue.

A market model with bid and ask price processes was mainly developed in the famous paper of Jouini and Kallal (1995) where the main result states that the socalled no free lunch (NFL) is equivalent to the existence of a CPS. Actually it was the first serious paper on arbitrage in markets with transaction costs. Note that the result of Grigoriev (2005) also strengthens the one of Jouini and Kallal (1995) in the case of one risky asset.

In our paper we consider a market with multi-dimensional bid and ask processes and with a money account, which corresponds to a model of stock market. In many papers on arbitrage with transaction costs also cited here, the authors consider models which refer to currency markets. Furthermore we deal with a classical notion of no-arbitrage without any additional assumptions. In most papers such an approach is uncommon due to difficulties arising from friction in markets. We introduce the notion of an equivalent bid-ask martingale measure (EBAMM) and prove that in a model with bid-ask spreads and an arbitrary state space $\Omega$, the existence of such a measure is equivalent to no-arbitrage. This measure seems to be natural and similar concepts can be found in the literature (see e.g. delta-martingale in Cherny 2007 and especially the concept of a $\kappa$-approximate martingale law in Dolinsky and Soner 2014). We also introduce the notions of a supermartingale consistent price system (supCPS) and a submartingale consistent price system (subCPS), and show that the existence of an EBAMM is equivalent to the existence of both such processes. This condition can be seen as a counterpart of the existence of a CPS, which is the main condition in many papers on this topic. 
The paper improves some of the results of Jouini and Kallal (1995) and Grigoriev (2005). We give necessary and sufficient conditions for the absence of arbitrage (e.g. instead of NFL or robust no-arbitrage), and we consider a general model of $d$ risky assets and a money account. Moreover, the notion of EBAMM can be seen as a generalization of an equivalent martingale measure (EMM) which is successfully used in markets without friction. It can also give an alternative to the concept of a CPS, which is a useful tool for pricing. It is a problem for future research how to apply EBAMM in the pricing of contingent claims.

The paper is organized as follows. In Sect. 2 a model of a financial market and some basic definitions are introduced. In Sect. 3 the main theorem is stated and proved. The last section gives some applications: mainly the Cox-Ross-Rubinstein model with bid-ask spreads is considered.

\section{A mathematical model of a financial market}

Let $(\Omega, \mathcal{F}, \mathbb{P})$ be a complete probability space equipped with a discrete-time filtration $\mathbb{F}=\left(\mathcal{F}_{t}\right)_{t=0}^{T}$ such that $\mathcal{F}_{T}=\mathcal{F}$ and $T$ is a finite time horizon. Assume that there are two processes $\underline{S}=\left(\underline{S}_{t}\right)_{t=0}^{T}=\left(\underline{S}_{t}^{1}, \ldots, \underline{S}_{t}^{d}\right)_{t=0}^{T}$ and $\bar{S}=\left(\bar{S}_{t}\right)_{t=0}^{T}=\left(\bar{S}_{t}^{1}, \ldots, \bar{S}_{t}^{d}\right)_{t=0}^{T}$, which are $d$-dimensional and adapted to $\mathbb{F}$. Furthermore we assume that $\underline{S}_{t}^{i} \leq \bar{S}_{t}^{i}$ for any $t=0,1, \ldots, T$ and $i=1, \ldots, d$. These processes model the prices of the assets: at every time $t$ the investor can buy or sell an unlimited number of the $i$-th assets at prices $\bar{S}_{t}^{i}$ and $\underline{S}_{t}^{i}$ respectively. We call $\underline{S}$ the bid price process and $\bar{S}$ the ask price process. The pair $(\underline{S}, \bar{S})$ will be called the bid-ask price process. Let us assume the existence of a money account or a bond, which is a strictly positive adapted (not necessarily predictable) process $B=\left(B_{t}\right)_{t=0}^{T}$ and all transactions are calculated in units of this process. For simplicity we assume that $B_{t} \equiv 1$ for all $t=0, \ldots, T$. This assumption does not restrict the generality of our model thanks to the discounting procedure, described in detail in Delbaen and Schachermayer (2006, Sect. 2.1) for markets without transaction costs. Note also that our model corresponds to a model of stock market rather than currency market.

A trading strategy is a $d$-dimensional process $H=\left(H_{t}\right)_{t=1}^{T}=\left(H_{t}^{1}, \ldots, H_{t}^{d}\right)_{t=1}^{T}$ which is predictable with respect to $\mathbb{F}$. We denote the set of all such strategies by $\mathcal{P}_{T}$. We also define its subsets $\mathcal{P}_{T}^{+}:=\left\{H \in \mathcal{P}_{T} \mid H \geq 0\right\}, \mathcal{P}_{T}^{-}:=\left\{H \in \mathcal{P}_{T} \mid H \leq 0\right\}$ where $H \geq 0$ iff $H^{i} \geq 0$ for any $i=1, \ldots, d$. We write $(H \cdot S)_{t}:=\sum_{j=1}^{t} H_{j} \cdot \Delta S_{j}$ where $\cdot$ is the inner product in $\mathbb{R}^{d}$.

Let $x=\left(x_{t}\right)_{t=1}^{T}$ be a value process in the market with bid-ask spreads for the strategy $H$ starting from 0 units in bank and stock accounts, i.e. $x_{t}$ is defined as follows:

$x_{t}=x_{t}(H):=-\sum_{j=1}^{t}\left(\Delta H_{j}\right)^{+} \cdot \bar{S}_{j-1}+\sum_{j=1}^{t}\left(\Delta H_{j}\right)^{-} \cdot \underline{S}_{j-1}+\left(H_{t}\right)^{+} \cdot \underline{S}_{t}-\left(H_{t}\right)^{-} \cdot \bar{S}_{t}$

where $\Delta H_{j}^{i}=H_{j}^{i}-H_{j-1}^{i}$ for any $i=1, \ldots, d$ and $j=1, \ldots, t$. In particular we set $\Delta H_{1}^{i}=H_{1}^{i}$ and we will usually skip the symbol of the inner product. The 
random variable $x_{t}$ models the gain or loss incurred up to time $t$. The first sum is the aggregate purchases of assets up to time $t$, while the second sum corresponds to the aggregate sales. Notice that at time $t$ we liquidate all positions in risky assets and we have $\sum_{j=1}^{t} \Delta H_{j}=H_{t}$. This can be interpreted as follows. If we want to know the real value of our portfolio at time $t$, we should calculate it in units of the money account as if we liquidated all positions in risky assets. In the literature this is known as the immediate liquidation value of the portfolio.

Remark 1 Notice that all changes in units of risky assets must be obtained by borrowing from or investing in the money account. Hence our position in the money account is uniquely determined by the strategy, which is actually self-financing.

We will use the notation $L^{0}\left(\mathbb{R}^{d}, \mathcal{F}_{t}\right)$ for the set of $\mathcal{F}_{t}$-measurable random vectors taking values in $\mathbb{R}^{d}$, with the convention that $L^{0}\left(\mathbb{R}^{d}\right)$ stands for $L^{0}\left(\mathbb{R}^{d}, \mathcal{F}_{T}\right)$. In the case of random variables (i.e. $d=1)$ we use the abbreviations $L^{0}\left(\mathcal{F}_{t}\right):=L^{0}\left(\mathbb{R}, \mathcal{F}_{t}\right)$ and $L^{0}:=L^{0}(\mathbb{R})$. Moreover let $L_{+}^{0}\left(\mathbb{R}^{d}, \mathcal{F}_{t}\right)$ denote the subspace of $L^{0}\left(\mathbb{R}^{d}, \mathcal{F}_{t}\right)$ consisting of all non-negative random vectors. To simplify the notation we will use the same convention as previously, i.e. we will write $L_{+}^{0}\left(\mathbb{R}^{d}\right), L_{+}^{0}\left(\mathcal{F}_{t}\right), L_{+}^{0}$ as appropriate. Furthermore the standard spaces $L^{1}$ and $L^{\infty}$ are treated in the same way.

To make our reasoning clearer we introduce for any $1 \leq t \leq t+k \leq T$ and $H \in L^{0}\left(\mathbb{R}^{d}, \mathcal{F}_{t-1}\right)$ the following random variable:

$$
x_{t-1, t+k}(H):=-(H)^{+} \cdot \bar{S}_{t-1}+(H)^{-} \cdot \underline{S}_{t-1}+(H)^{+} \cdot \underline{S}_{t+k}-(H)^{-} \cdot \bar{S}_{t+k} .
$$

Now set $\mathcal{R}_{T}:=\left\{x_{T}(H) \mid H \in \mathcal{P}_{T}\right\}$, and define the set of hedgeable claims to be

$$
\mathcal{A}_{T}:=\mathcal{R}_{T}-L_{+}^{0}
$$

Let $\overline{\mathcal{A}}_{T}$ denote the closure of $\mathcal{A}_{T}$ in probability. The following definition is crucial.

Definition 1 We say that there is no arbitrage in the market with bid-ask spreads if

$$
\mathcal{R}_{T} \cap L_{+}^{0}=\{0\}
$$

Notice that condition (NA) is equivalent to $\mathcal{A}_{T} \cap L_{+}^{0}=\{0\}$. Now we introduce the counterparts of the set $\mathcal{R}_{T}$ in the simplified situation when we can only buy or short sale assets between two time moments. Define, for any $0 \leq j<t \leq T$,

$$
\begin{aligned}
& \mathcal{R}_{j, t}^{+}:=\left\{H \cdot\left(\underline{S}_{t}-\bar{S}_{j}\right) \mid H \in L_{+}^{0}\left(\mathbb{R}^{d}, \mathcal{F}_{j}\right)\right\}, \\
& \mathcal{R}_{j, t}^{-}:=\left\{H \cdot\left(\bar{S}_{t}-\underline{S}_{j}\right) \mid-H \in L_{+}^{0}\left(\mathbb{R}^{d}, \mathcal{F}_{j}\right)\right\} .
\end{aligned}
$$

Furthermore for any $1 \leq t \leq t+k \leq T$ set

$$
\mathbb{F}_{t-1, t+k}:=\mathcal{R}_{t-1, t+k}^{+}+\mathcal{R}_{t-1, t+k}^{-}, \quad F_{t-1, t+k}:=\mathbb{F}_{t-1, t+k}-L_{+}^{0}\left(\mathcal{F}_{t+k}\right) .
$$


As counterparts of $\mathcal{R}_{t}$ and $\mathcal{A}_{t}$, for any $t=1, \ldots, T$, we set

$$
\mathbb{F}_{t}:=\sum_{j<t} \mathcal{R}_{j, t}^{+}+\sum_{j<t} \mathcal{R}_{j, t}^{-} \text {and } F_{t}:=\mathbb{F}_{t}-L_{+}^{0}\left(\mathcal{F}_{t}\right)
$$

Consequently, we also introduce $\Lambda_{T}:=\sum_{t=1}^{T} \mathbb{F}_{t}-L_{+}^{0}$.

Remark 2 Notice that $F_{t-1, t+k}, \mathbb{F}_{t-1, t+k}, \mathbb{F}_{t}, F_{t}$ and $\Lambda_{t}$ are convex cones and $\Lambda_{T}=$ $\sum_{t=1}^{T} F_{t}$.

Lemma 1 Assume that $\mathcal{A}_{T} \cap L_{+}^{0}=\{0\}$. Then $\mathcal{A}_{t} \cap L_{+}^{0}\left(\mathcal{F}_{t}\right)=\{0\}$ for any $t<T$.

Proof Notice that if $H$ is an arbitrage strategy in a model with time horizon $t$ (so at time $t$ we liquidate all positions in stock), then it is also an arbitrage strategy in a model with any larger time horizon, in particular with time horizon $T$ : it suffices to take the same strategy $H$ up to time $t$ and later 0 .

We now introduce the definition of a CPS, similar to the one in Jouini and Kallal (1995).

Definition 2 We say that a pair $(\tilde{S}, \tilde{P})$ is a consistent price system (CPS) in the market with bid-ask spreads when $\tilde{P}$ is a probability measure equivalent to $\mathbb{P}$ and $\tilde{S}=\left(\tilde{S}_{t}\right)_{t=0}^{T}$ is a $d$-dimensional process adapted to the filtration $\mathbb{F}$, which is a $\tilde{P}$-martingale and

$$
\underline{S}_{t}^{i} \leq \tilde{S}_{t}^{i} \leq \bar{S}_{t}^{i} \mathbb{P} \text {-a.e. }
$$

for all $i=1, \ldots, d$ and $t=0, \ldots, T$.

If the process $\tilde{S}$ is a $\tilde{P}$-supermartingale (resp. $\tilde{P}$-submartingale) then we say that the pair $(\tilde{S}, \tilde{P})$ is a supermartingale consistent price system (supCPS) [(resp. a submartingale consistent price system (subCPS)].

We introduce the notion of EBAMM, which will play a similar role to an EMM in markets without friction.

Definition 3 We shall say that a probability measure $\mathbb{Q}$ is an equivalent bid-ask martingale measure $(\mathrm{EBAMM})$ for the bid-ask price process $(\underline{S}, \bar{S})$ if $\mathbb{Q} \sim \mathbb{P}$, all $\underline{S}_{t}, \bar{S}_{t} \in L^{1}(\mathbb{Q})$, and

$$
\underline{S}_{t-1}^{i} \leq E_{\mathbb{Q}}\left(\bar{S}_{t}^{i} \mid \mathcal{F}_{t-1}\right) \quad \text { and } \quad E_{\mathbb{Q}}\left(\underline{S}_{t}^{i} \mid \mathcal{F}_{t-1}\right) \leq \bar{S}_{t-1}^{i}, \quad \mathbb{P} \text {-a.e. }
$$

for any $t=1, \ldots, T$ and $i=1, \ldots, d$.

The interpretation of this measure is quite obvious. Let us consider an EBAMM in the context of stock market. If we buy shares at any time $t-1$ at price $\bar{S}_{t-1}^{i}$, we should not expect, on average, that at time $t$ we shall sell them at a better price. Of course, "average" here is understood as a conditional expectation with respect to $\mathbb{Q}$. The analogous situation occurs if we short sale shares. The following lemma presents the straightforward relation between CPS and EBAMM. 
Lemma 2 If there exists a CPS, then there exists an EBAMM.

Proof Let $(\tilde{S}, \mathbb{Q})$ be a CPS. Then for any $t=1, \ldots, T$ and $i=1, \ldots, d$ we have

$$
\begin{gathered}
E_{\mathbb{Q}}\left(\underline{S}_{t}^{i} \mid \mathcal{F}_{t-1}\right) \leq E_{\mathbb{Q}}\left(\tilde{S}_{t}^{i} \mid \mathcal{F}_{t-1}\right)=\tilde{S}_{t-1}^{i} \leq \bar{S}_{t-1}^{i}, \\
\underline{S}_{t-1}^{i} \leq \tilde{S}_{t-1}^{i}=E_{\mathbb{Q}}\left(\tilde{S}_{t}^{i} \mid \mathcal{F}_{t-1}\right) \leq E_{\mathbb{Q}}\left(\bar{S}_{t}^{i} \mid \mathcal{F}_{t-1}\right) .
\end{gathered}
$$

Remark 3 The notion of EBAMM can be seen as a generalization of an EMM in markets without friction. Indeed, when we assume that $\underline{S}=\bar{S}$, then our model comes down to a finite discrete time market model without transaction costs, and EBAMM is actually the same as EMM.

\section{Main results}

First we give a sufficient condition for the absence of arbitrage, which is actually the existence of a CPS. This result is standard and well-known but we will prove it in our model using a slightly weaker assumption. Set

$$
\begin{aligned}
\tilde{R}_{T} & :=\left\{(\hat{H} \cdot \hat{S})_{T}+(\check{H} \cdot \check{S})_{T} \mid \hat{H} \in \mathcal{P}_{T}^{+}, \check{H} \in \mathcal{P}_{T}^{-}\right\} \\
\tilde{R}_{T}^{b} & :=\left\{(\hat{H} \cdot \hat{S})_{T}+(\check{H} \cdot \check{S})_{T} \mid \hat{H} \in \mathcal{P}_{T}^{+}, \check{H} \in \mathcal{P}_{T}^{-} \text {where } \hat{H}, \check{H} \text { are bounded } b i g\right\} .
\end{aligned}
$$

We prove two auxiliary lemmas.

Lemma 3 The following conditions are equivalent:

(a) $\tilde{R}_{T} \cap L_{+}^{0}=\{0\}$;

(b) $\left\{\hat{\eta} \Delta \hat{S}_{t}+\check{\eta} \Delta \check{S}_{t}: \hat{\eta},-\check{\eta} \in L_{+}^{0}\left(\mathbb{R}^{d}, \mathcal{F}_{t-1}\right)\right\} \cap L_{0}^{+}\left(\mathcal{F}_{t}\right)=\{0\}$ for any $t=1, \ldots, T$.

Proof The implication (a) $\Rightarrow$ (b) is obvious. To show (b) $\Rightarrow$ (a), we reason as in Kabanov and Safarian (2009, Sect. 2.1.1). Assume that we have the absence of arbitrage in any one-step model and suppose that $\tilde{R}_{T} \cap L_{+}^{0} \neq\{0\}$. Take the smallest $t \leq T$ such that $\tilde{R}_{t} \cap L_{+}^{0}\left(\mathcal{F}_{t}\right) \neq\{0\}$ and notice that $t>1$. Hence there exist strategies $\hat{H} \in \mathcal{P}_{t}^{+}$, $\check{H} \in \mathcal{P}_{t}^{-}$such that

$$
(\hat{H} \cdot \hat{S})_{t}+(\check{H} \cdot \check{S})_{t} \geq 0 \text { and } \mathbb{P}\left((\hat{H} \cdot \hat{S})_{t}+(\check{H} \cdot \check{S})_{t}>0\right)>0
$$

Due to the choice of $t$, either the set $\Gamma^{\prime}:=\left\{(\hat{H} \cdot \hat{S})_{t-1}+(\check{H} \cdot \check{S})_{t-1}<0\right\}$ is of strictly positive probability (we set $\hat{\eta}:=\mathbb{1}_{\Gamma^{\prime}} \hat{H}_{t}, \check{\eta}:=\mathbb{1}_{\Gamma^{\prime}} \check{H}_{t}$ ), or the set $\Gamma^{\prime \prime}:=$ $\left\{(\hat{H} \cdot \hat{S})_{t-1}+(\check{H} \cdot \check{S})_{t-1}=0\right\}$ is of full measure (we take $\hat{\eta}:=\mathbb{1}_{\Gamma^{\prime \prime}} \hat{H}_{t}, \check{\eta}:=\mathbb{1}_{\Gamma^{\prime \prime}} \check{H}_{t}$ ). In any case we have a contradiction.

Lemma 4 The following conditions are equivalent: 
(a) $\tilde{R}_{T} \cap L_{+}^{0}=\{0\}$;

(b) $\tilde{R}_{T}^{b} \cap L_{+}^{0}=\{0\}$.

Proof The implication (a) $\Rightarrow$ (b) is trivial. Now assume that $\tilde{R}_{T}^{b} \cap L_{+}^{0}=\{0\}$. To show that $\tilde{R}_{T} \cap L_{+}^{0}=\{0\}$, by Lemma 3 it suffices to prove that

$$
\left\{\hat{\eta} \Delta \hat{S}_{t}+\check{\eta} \Delta \check{S}_{t}: \hat{\eta},-\check{\eta} \in L_{+}^{0}\left(\mathbb{R}^{d}, \mathcal{F}_{t-1}\right)\right\} \cap L_{0}^{+}\left(\mathcal{F}_{t}\right)=\{0\} \quad \text { for any } t=1, \ldots, T \text {. }
$$

Fix any $t$ and suppose that there exist random variables $\hat{H}_{t} \in L_{+}^{0}\left(\mathbb{R}^{d}, \mathcal{F}_{t-1}\right),-\check{H}_{t} \in$ $L_{+}^{0}\left(\mathbb{R}^{d}, \mathcal{F}_{t-1}\right)$ satisfying

$$
\hat{H}_{t} \Delta \hat{S}_{t}+\check{H}_{t} \Delta \check{S}_{t} \geq 0 \mathbb{P} \text {-a.e. and } \mathbb{P}\left(\hat{H}_{t} \Delta \hat{S}_{t}+\check{H}_{t} \Delta \check{S}_{t}>0\right)>0 \text {. }
$$

Define $H_{t}:=\left(\hat{H}_{t}, \check{H}_{t}\right) \in L^{0}\left(\mathbb{R}^{2 d}, \mathcal{F}_{t-1}\right)$ and consider the normalized random vector

$$
\bar{H}_{t}:= \begin{cases}H_{t} /\left\|H_{t}\right\|, & H_{t} \neq 0 \\ 0, & H_{t}=0\end{cases}
$$

We set $\hat{H}_{t}^{b}:=\left(\bar{H}_{t}^{1}, \ldots, \bar{H}_{t}^{d}\right)$ and $\check{H}_{t}^{b}:=\left(\bar{H}_{t}^{d+1}, \ldots, \bar{H}_{t}^{2 d}\right)$. Notice that $\hat{H}_{t}^{b} \in$ $L_{+}^{0}\left(\mathbb{R}^{d}, \mathcal{F}_{t-1}\right),-\check{H}_{t}^{b} \in L_{+}^{0}\left(\mathbb{R}^{d}, \mathcal{F}_{t-1}\right)$, and

$$
\hat{H}_{t}^{b} \Delta \hat{S}_{t}+\check{H}_{t}^{b} \Delta \check{S}_{t} \geq 0 \quad \mathbb{P} \text {-a.e. and } \mathbb{P}\left(\hat{H}_{t}^{b} \Delta \hat{S}_{t}+\check{H}_{t}^{b} \Delta \check{S}_{t}>0\right)>0
$$

This is a one-step arbitrage in the class of bounded strategies, contradicting $\tilde{R}_{T}^{b} \cap L_{+}^{0}=$ \{0\} [We may also use the arguments of Kabanov and Safarian (2009, Sect. 2.1.1). Define $H_{t}^{n}:=H_{t} \mathbb{1}_{\left\{\left\|H_{t}\right\| \leq n\right\}}$. Then there exists a sufficiently large $n \in \mathbb{N}$ such that $H_{t}^{n}$ satisfies (2)].

Theorem 1 Assume that there exists a supCPS $(\hat{S}, \mathbb{Q})$ and a subCPS $(\check{S}, \mathbb{Q})$. Then $\tilde{R}_{T} \cap L_{+}^{0}=\{0\}$ and there is no arbitrage, i.e. $\mathcal{A}_{T} \cap L_{+}^{0}=\{0\}$.

Proof By Lemma 4 it suffices to prove that $\tilde{R}_{T}^{b} \cap L_{+}^{0}=\{0\}$. Take any $X=(\hat{H}$. $\hat{S})_{T}+(\check{H} \cdot \check{S})_{T} \in \tilde{R}_{T}^{b} \cap L_{+}^{0}$. Hence $(\hat{H} \cdot \hat{S})_{T}+(\check{H} \cdot \check{S})_{T} \geq 0$ and in particular $H$ is a bounded strategy. We show that $E_{\mathbb{Q}}\left[(\hat{H} \cdot \hat{S})_{T}+(\check{H} \cdot \check{S})_{T}\right] \leq 0$. Using the assumption that $\hat{S}$ is a $\mathbb{Q}$-supermartingale and $\check{S}$ a $\mathbb{Q}$-submartingale we get $E_{\mathbb{Q}}\left(\hat{H}_{t} \Delta \hat{S}_{t} \mid \mathcal{F}_{t-1}\right)=$ $\hat{H}_{t} E_{\mathbb{Q}}\left(\Delta \hat{S}_{t} \mid \mathcal{F}_{t-1}\right) \leq 0$. Analogously $E_{\mathbb{Q}}\left(\check{H}_{t} \Delta \check{S}_{t} \mid \mathcal{F}_{t-1}\right) \leq 0$. Summing up,

$$
E_{\mathbb{Q}}\left[(\hat{H} \cdot \hat{S})_{T}+(\check{H} \cdot \check{S})_{T}\right] \leq 0 .
$$

Hence $X=0 \mathbb{Q}$-a.e. and from the equivalence of measures, $X=0 \mathbb{P}$-a.e. We show now that $\mathcal{A}_{T} \cap L_{+}^{0}=\{0\}$. Take any $\xi \in \mathcal{A}_{T} \cap L_{+}^{0}$. Then

$$
0 \leq \xi \leq-\sum_{t=1}^{T}\left(\Delta H_{t}\right)^{+} \bar{S}_{t-1}+\sum_{t=1}^{T}\left(\Delta H_{t}\right)^{-} \underline{S}_{t-1}+\left(H_{T}\right)^{+} \underline{S}_{T}-\left(H_{T}\right)^{-} \bar{S}_{T} .
$$


Notice that for any strategy $H \in \mathcal{P}_{T}$ there exist $\hat{H} \in \mathcal{P}_{T}^{+}$and $\check{H} \in \mathcal{P}_{T}^{-}$such that $\Delta H_{t}^{i}=\Delta \hat{H}_{t}^{i}+\Delta \check{H}_{t}^{i}$. We can define them as follows:

$$
\hat{H}_{t}^{i}:=\left(H_{t}^{i}\right)^{+} \text {and } \check{H}_{t}^{i}:=-\left(H_{t}^{i}\right)^{-} \text {for any } t=1, \ldots, T, i=1, \ldots, d \text {. }
$$

This means that we split the strategy into two strategies, which consist of long and short positions only. Moreover, notice that the set $\left\{\Delta \hat{H}_{t}^{i}>0, \Delta \check{H}_{t}^{i}<0\right\}=\emptyset \mathbb{P}$-a.e. Indeed, otherwise $\left(H_{t}^{i}\right)^{+}>\left(H_{t-1}^{i}\right)^{+} \geq 0$ and $\left(H_{t}^{i}\right)^{-}>\left(H_{t-1}^{i}\right)^{-} \geq 0$ on the same set of positive measure, a contradiction. Analogously we get $\left\{\Delta \hat{H}_{t}^{i}<0, \Delta \check{H}_{t}^{i}>0\right\}=\emptyset \mathbb{P}$ a.e. Hence, we have the following implications for any $t=1, \ldots, T$ and $i=1, \ldots, d$. If $\Delta H_{t}^{i}>0$ then $\Delta \hat{H}_{t}^{i}, \Delta \check{H}_{t}^{i}>0$ and if $\Delta H_{t}^{i}<0$ then $\Delta \hat{H}_{t}^{i}, \Delta \check{H}_{t}^{i}<0$. Hence we always have $\left(\Delta H_{t}^{i}\right)^{+}=\left(\Delta \hat{H}_{t}^{i}\right)^{+}+\left(\Delta \check{H}_{t}^{i}\right)^{+}$as well as $\left(\Delta H_{t}^{i}\right)^{-}=\left(\Delta \hat{H}_{t}^{i}\right)^{-}+\left(\Delta \check{H}_{t}^{i}\right)^{-}$. Summing up, we get

$$
\begin{aligned}
\xi \leq & -\sum_{t=1}^{T}\left(\Delta H_{t}\right)^{+} \bar{S}_{t-1}+\sum_{t=1}^{T}\left(\Delta H_{t}\right)^{-} \underline{S}_{t-1}+\left(H_{T}\right)^{+} \underline{S}_{T}-\left(H_{T}\right)^{-} \bar{S}_{T} \\
= & -\sum_{t=1}^{T}\left(\Delta \hat{H}_{t}\right)^{+} \bar{S}_{t-1}+\sum_{t=1}^{T}\left(\Delta \hat{H}_{t}\right)^{-} \underline{S}_{t-1}+\left(\hat{H}_{T}\right)^{+} \underline{S}_{T}-\left(\hat{H}_{T}\right)^{-} \bar{S}_{T} \\
& -\sum_{t=1}^{T}\left(\Delta \check{H}_{t}\right)^{+} \bar{S}_{t-1}+\sum_{t=1}^{T}\left(\Delta \check{H}_{t}\right)^{-} \underline{S}_{t-1}+\left(\check{H}_{T}\right)^{+} \underline{S}_{T}-\left(\check{H}_{T}\right)^{-} \bar{S}_{T}=:(\star)
\end{aligned}
$$

Notice that

$$
\underline{S}_{t}^{i} \leq \hat{S}_{t}^{i} \leq \bar{S}_{t}^{i} \quad \text { and } \quad \underline{S}_{t}^{i} \leq \check{S}_{t}^{i} \leq \bar{S}_{t}^{i}, \quad \mathbb{P} \text {-a.e. }
$$

for any $t=0, \ldots, T$ and $i=1, \ldots, d$. Hence

$$
\begin{aligned}
(\star) \leq & -\sum_{t=1}^{T}\left(\Delta \hat{H}_{t}\right)^{+} \hat{S}_{t-1}+\sum_{t=1}^{T}\left(\Delta \hat{H}_{t}\right)^{-} \hat{S}_{t-1}+\left(\hat{H}_{T}\right)^{+} \hat{S}_{T}-\left(\hat{H}_{T}\right)^{-} \hat{S}_{T} \\
& -\sum_{t=1}^{T}\left(\Delta \check{H}_{t}\right)^{+} \check{S}_{t-1}+\sum_{t=1}^{T}\left(\Delta \check{H}_{t}\right)^{-} \check{S}_{t-1}+\left(\check{H}_{T}\right)^{+} \check{S}_{T}-\left(\check{H}_{T}\right)^{-} \check{S}_{T} \\
= & -\sum_{t=1}^{T} \Delta \hat{H}_{t} \hat{S}_{t-1}+\hat{H}_{T} \hat{S}_{T}-\sum_{t=1}^{T} \Delta \check{H}_{t} \check{S}_{t-1}+\check{H}_{T} \check{S}_{T}=(\hat{H} \cdot \hat{S})_{T}+(\check{H} \cdot \check{S})_{T} .
\end{aligned}
$$

Then

$$
0 \leq \xi \leq(\hat{H} \cdot \hat{S})_{T}+(\check{H} \cdot \check{S})_{T} .
$$

From $\tilde{R}_{T} \cap L_{+}^{0}=\{0\}$ we get $(\hat{H} \cdot \hat{S})_{T}+(\check{H} \cdot \check{S})_{T}=0 \mathbb{P}$-a.e. and hence $\xi=0$ $\mathbb{P}$-a.e. 
Remark 4 Notice that if there exists a CPS then the assumptions of Theorem 1 are also satisfied and we have the absence of arbitrage, i.e. $\mathcal{A}_{T} \cap L_{+}^{0}=\{0\}$.

Remark 5 The strategies $\hat{H} \in \mathcal{P}_{T}^{+}$and $\check{H} \in \mathcal{P}_{T}^{-}$in the proof of Theorem 1 can also be constructed in the following way:

$$
\begin{aligned}
& \text { If } H_{t}^{i} \geq 0 \text { on }\left\{H_{t-1}^{i} \geq 0\right\} \text { then } \Delta \hat{H}_{t}^{i}:=\Delta H_{t}^{i}, \Delta \check{H}_{t}^{i}:=0, \\
& \text { if } H_{t}^{i}<0 \text { on }\left\{H_{t-1}^{i}<0\right\} \text { then } \Delta \hat{H}_{t}^{i}:=0, \Delta \check{H}_{t}^{i}:=\Delta H_{t}^{i}, \\
& \text { if } H_{t}^{i} \geq 0 \text { on }\left\{H_{t-1}^{i}<0\right\} \text { then } \Delta \hat{H}_{t}^{i}:=H_{t}^{i}, \Delta \check{H}_{t}^{i}:=-H_{t-1}^{i}, \\
& \text { if } H_{t}^{i}<0 \text { on }\left\{H_{t-1}^{i} \geq 0\right\} \text { then } \Delta \hat{H}_{t}^{i}:=-H_{t-1}^{i}, \Delta \check{H}_{t}^{i}:=H_{t}^{i} .
\end{aligned}
$$

By induction we can show that these are actually the same strategies.

Before we formulate the fundamental theorem, we prove some technical lemmas.

Lemma 5 Let $\Pi \in \mathbb{F}_{t}$. Then there exist predictable and non-negative, d-dimensional processes $\vartheta=\left(\vartheta_{j}\right)_{j=1}^{t}, \tilde{\vartheta}=\left(\tilde{\vartheta}_{j}\right)_{j=1}^{t}$ such that for any $j=1, \ldots, t$ and $i=1, \ldots, d$ we have $\left\{\vartheta_{j}^{i}>0, \tilde{\vartheta}_{j}^{i}>0\right\}=\emptyset \mathbb{P}$-a.e. and

$$
\Pi \leq \Xi:=-\sum_{j=1}^{t} \vartheta_{j} \bar{S}_{j-1}+\sum_{j=1}^{t} \tilde{\vartheta}_{j} \underline{S}_{j-1}+\sum_{j=1}^{t} \vartheta_{j} \underline{S}_{t}-\sum_{j=1}^{t} \tilde{\vartheta}_{j} \bar{S}_{t} \quad \mathbb{P} \text {-a.e. }
$$

Proof Without loss of generality we can assume that $\Pi$ is of the form

$$
\Pi=-\sum_{j=1}^{t} \theta_{j} \bar{S}_{j-1}+\sum_{j=1}^{t} \tilde{\theta}_{j} \underline{S}_{j-1}+\sum_{j=1}^{t} \theta_{j} \underline{S}_{t}-\sum_{j=1}^{t} \tilde{\theta}_{j} \bar{S}_{t}
$$

where $\Theta=\left(\theta_{j}\right)_{j=1}^{t}, \tilde{\Theta}=\left(\tilde{\theta}_{j}\right)_{j=1}^{t}$ are predictable and non-negative, $d$-dimensional processes. Let $v_{j}^{i}:=\min \left\{\theta_{j}^{i}, \tilde{\theta}_{j}^{i}\right\} \geq 0$. We define $\vartheta, \tilde{\vartheta}$ as follows:

$$
\vartheta_{j}^{i}:=\theta_{j}^{i}-v_{j}^{i}, \quad \tilde{\vartheta}_{j}^{i}:=\tilde{\theta}_{j}^{i}-v_{j}^{i} \text { for } i=1, \ldots, d, j=1, \ldots, t .
$$

Notice that $\vartheta, \tilde{\vartheta}$ are predictable and non-negative, $d$-dimensional processes. We have

$$
\begin{aligned}
\Pi & =-\sum_{j=1}^{t}\left(\vartheta_{j}+v_{j}\right) \bar{S}_{j-1}+\sum_{j=1}^{t}\left(\tilde{\vartheta}_{j}+v_{j}\right) \underline{S}_{j-1}+\sum_{j=1}^{t}\left(\vartheta_{j}+v_{j}\right) \underline{S}_{t}-\sum_{j=1}^{t}\left(\tilde{\vartheta}_{j}+v_{j}\right) \bar{S}_{t} \\
& =\Xi-\sum_{j=1}^{t} v_{j}\left(\bar{S}_{j-1}-\underline{S}_{j-1}\right)-\sum_{j=1}^{t} v_{j}\left(\bar{S}_{t}-\underline{S}_{t}\right) \leq \Xi
\end{aligned}
$$

because the last two sums are non-negative. 
Lemma 6 For any $t=1, \ldots, T$ we have $F_{t} \subset \mathcal{A}_{t}$.

Proof It suffices to show that $\mathbb{F}_{t} \subset \mathcal{A}_{t}$ where $\mathbb{F}_{t}$ is as in (1). Take any $\Pi \in \mathbb{F}_{t}$. By Lemma 5 there exist predictable and non-negative, $d$-dimensional processes $\vartheta=$ $\left(\vartheta_{j}\right)_{j=1}^{t}, \tilde{\vartheta}=\left(\tilde{\vartheta}_{j}\right)_{j=1}^{t}$ such that for any $j=1, \ldots, t$ and $i=1, \ldots, d$ we have $\left\{\vartheta_{j}^{i}>0, \tilde{\vartheta}_{j}^{i}>0\right\}=\emptyset \mathbb{P}$-a.e. and

$$
\Pi \leq \Xi:=-\sum_{j=1}^{t} \vartheta_{j} \bar{S}_{j-1}+\sum_{j=1}^{t} \tilde{\vartheta}_{j} \underline{S}_{j-1}+\sum_{j=1}^{t} \vartheta_{j} \underline{S}_{t}-\sum_{j=1}^{t} \tilde{\vartheta}_{j} \bar{S}_{t} \quad \mathbb{P} \text {-a.e. }
$$

Define the strategy $H=\left(H_{j}\right)_{j=1}^{t} \in \mathcal{P}_{t}$ as follows:

$$
\Delta H_{j}:=\left(\Delta H_{j}\right)^{+}-\left(\Delta H_{j}\right)^{-} \text {where }\left(\Delta H_{j}\right)^{+}:=\vartheta_{j} \quad \text { and } \quad\left(\Delta H_{j}\right)^{-}:=\tilde{\vartheta}_{j}
$$

Moreover, we set $H_{1}:=\Delta H_{1}$ and $H_{j}:=\Delta H_{j}+H_{j-1}$ for $j>1$. Notice that $H$ is a well defined strategy. Furthermore

$$
\begin{aligned}
& \sum_{j=1}^{t}\left(\Delta H_{j}\right)^{-} \bar{S}_{t}-\sum_{j=1}^{t}\left(\Delta H_{j}\right)^{+} \underline{S}_{t}+H_{t}^{+} \underline{S}_{t}-H_{t}^{-} \bar{S}_{t} \\
& \quad=\left(H_{t}^{+}-\sum_{j=1}^{t}\left(\Delta H_{j}\right)^{+}\right) \underline{S}_{t}-\left(H_{t}^{-}-\sum_{j=1}^{t}\left(\Delta H_{j}\right)^{-}\right) \bar{S}_{t}
\end{aligned}
$$

and

$$
\sum_{j=1}^{t}\left(\Delta H_{j}\right)^{+}-\sum_{j=1}^{t}\left(\Delta H_{j}\right)^{-}=\sum_{j=1}^{t} \Delta H_{j}=H_{t}=H_{t}^{+}-H_{t}^{-}
$$

Therefore $H_{t}^{+}-\sum_{j=1}^{t}\left(\Delta H_{j}\right)^{+}=H_{t}^{-}-\sum_{j=1}^{t}\left(\Delta H_{j}\right)^{-}$and $H_{t}^{+} \leq \sum_{j=1}^{t}\left(\Delta H_{j}\right)^{+}$. Define $r:=\left(H_{t}^{+}-\sum_{j=1}^{t}\left(\Delta H_{j}\right)^{+}\right) \underline{S}_{t}-\left(H_{t}^{-}-\sum_{j=1}^{t}\left(\Delta H_{j}\right)^{-}\right) \bar{S}_{t}$. By the previous observation, $r=\left(H_{t}^{+}-\sum_{j=1}^{t}\left(\Delta H_{j}\right)^{+}\right)\left(\underline{S}_{t}-\bar{S}_{t}\right) \geq 0$, which simply means that $r \in L_{+}^{0}\left(\mathcal{F}_{t}\right)$. Hence

$$
\Pi+r \leq \Xi+r=-\sum_{j=1}^{t}\left(\Delta H_{j}\right)^{+} \bar{S}_{j-1}+\sum_{j=1}^{t}\left(\Delta H_{j}\right)^{-} \underline{S}_{j-1}+\left(H_{t}\right)^{+} \underline{S}_{t}-\left(H_{t}\right)^{-} \bar{S}_{t} .
$$

Obviously $\Xi+r=x_{t}(H) \in \mathcal{R}_{t}$ and $\Pi \leq x_{t}(H)-r$. Furthermore there exists $\tilde{r} \in L_{+}^{0}\left(\mathcal{F}_{t}\right)$ such that $\Pi=x_{t}(H)-r-\tilde{r}$. It suffices to define $\tilde{r}:=\Xi-\Pi$. Hence $\Pi \in \mathcal{A}_{t}$. 
Remark 6 It is not clear whether $\Lambda_{T} \subset \mathcal{A}_{T}$ or not. We only know that $\Lambda_{T} \subset \sum_{t=1}^{T} \mathcal{A}_{t}$.

Remark 7 Notice that for any $\Pi \in F_{T}$ there exists a strategy $H \in \mathcal{P}_{T}$ and a random variable $r \in L_{+}^{0}$ such that $\Pi=x_{T}(H)-r$.

Lemma 7 For any $1 \leq t \leq t+k \leq T$ and $x \in F_{t-1, t+k}$ there exist $H_{t} \in$ $L^{0}\left(\mathbb{R}^{d}, \mathcal{F}_{t-1}\right)$ and $r \in L_{+}^{0}\left(\mathcal{F}_{t+k}\right)$ such that $x=x_{t-1, t+k}\left(H_{t}\right)-r$.

Proof Fix $t, k$ such that $1 \leq t \leq t+k \leq T$ and take any $x \in F_{t-1, t+k}$. Let $x=\Pi-l$ where

$$
\Pi=-\theta \bar{S}_{t-1}+\tilde{\theta} \underline{S}_{t-1}+\theta \underline{S}_{t+k}-\tilde{\theta} \bar{S}_{t+k}
$$

and $\theta, \tilde{\theta} \in L_{+}^{0}\left(\mathbb{R}^{d}, \mathcal{F}_{t-1}\right), l \in L_{+}^{0}\left(\mathcal{F}_{t+k}\right)$. Notice that there exist $\vartheta, \tilde{\vartheta} \in$ $L_{+}^{0}\left(\mathbb{R}^{d}, \mathcal{F}_{t-1}\right)$ such that $\left\{\vartheta^{i}>0, \tilde{\vartheta}^{i}>0\right\}=\emptyset \mathbb{P}$-a.e. for any $i=1, \ldots, d$. It suffices to use the reasoning from the proof of Lemma 5. Let $v^{i}:=\min \left\{\theta^{i}, \tilde{\theta}^{i}\right\} \geq 0$. We define $\vartheta, \tilde{\vartheta}$ as follows:

$$
\vartheta^{i}:=\theta^{i}-v^{i}, \quad \tilde{\vartheta}^{i}:=\tilde{\theta}^{i}-v^{i} \text { for any } i=1, \ldots, d .
$$

Notice that $\vartheta, \tilde{\vartheta}$ are non-negative and $\mathcal{F}_{t-1}$-measurable, $d$-dimensional random vectors. Furthermore

$$
\begin{aligned}
\Pi & =-(\vartheta+v) \bar{S}_{t-1}+(\tilde{\vartheta}+v) \underline{S}_{t-1}+(\vartheta+v) \underline{S}_{t+k}-(\tilde{\vartheta}+v) \bar{S}_{t+k} \\
& =-\vartheta \bar{S}_{t-1}+\tilde{\vartheta} \underline{S}_{t-1}+\vartheta \underline{S}_{t+k}-\tilde{\vartheta} \bar{S}_{t+k}-v\left(\bar{S}_{t-1}-\underline{S}_{t-1}\right)-v\left(\bar{S}_{t+k}-\underline{S}_{t+k}\right) \\
& \leq \Xi:=-\vartheta \bar{S}_{t-1}+\tilde{\vartheta} \underline{S}_{t-1}+\vartheta \underline{S}_{t+k}-\tilde{\vartheta} \bar{S}_{t+k} \in \mathcal{R}_{t+k} \quad \mathbb{P} \text {-a.e. }
\end{aligned}
$$

Now define $H_{t}:=\vartheta-\tilde{\vartheta}$. Notice that $H_{t} \in L^{0}\left(\mathbb{R}^{d}, \mathcal{F}_{t-1}\right)$ and $\left(H_{t}^{i}\right)^{+}=\vartheta^{i},\left(H_{t}^{i}\right)^{-}=$ $\tilde{\vartheta}^{i}$. Moreover, $\tilde{l}:=\Xi-\Pi \in L_{+}^{0}\left(\mathcal{F}_{t+k}\right)$ and $x=\Pi-l=\Xi-l-\tilde{l}$. Let $r:=l+\tilde{l} \in$ $L_{+}^{0}\left(\mathcal{F}_{t+k}\right)$. Then

$$
\begin{aligned}
x & =-\left(H_{t}\right)^{+} \cdot \bar{S}_{t-1}+\left(H_{t}\right)^{-} \cdot \underline{S}_{t-1}+\left(H_{t}\right)^{+} \cdot \underline{S}_{t+k}-\left(H_{t}\right)^{-} \cdot \bar{S}_{t+k}-r \\
& =x_{t-1, t+k}\left(H_{t}\right)-r .
\end{aligned}
$$

The following theorem is the main result of the paper. It presents the equivalent conditions for the absence of arbitrage in markets with bid-ask spreads and a money account. 
Theorem 2 (Fundamental theorem) The following conditions are equivalent:

(a) $\mathcal{A}_{T} \cap L_{+}^{0}=\{0\}(N A)$;

(b) $F_{t} \cap L_{+}^{0}\left(\mathcal{F}_{t}\right)=\{0\}$ for any $t=1, \ldots, T$;

(c) $F_{t-1, t+k} \cap L_{+}^{0}\left(\mathcal{F}_{t+k}\right)=\{0\}$ for any $1 \leq t \leq t+k \leq T$;

(d) $F_{t-1, t+k} \cap L_{+}^{0}\left(\mathcal{F}_{t+k}\right)=\{0\}$ and $F_{t-1, t+k}=\bar{F}_{t-1, t+k}$ for any $1 \leq t \leq t+k \leq T$;

(e) $\bar{F}_{t-1, t+k} \cap L_{+}^{0}\left(\mathcal{F}_{t+k}\right)=\{0\}$ for any $1 \leq t \leq t+k \leq T$;

(f) there exists an EBAMM $\mathbb{Q}$ for the bid-ask process $(\underline{S}, \bar{S})$ such that $\frac{d \mathbb{Q}}{d \mathbb{P}} \in L^{\infty}$ (EBAMM);

(g) there exists a supCPS $(\hat{S}, \mathbb{Q})$ and a $\operatorname{subCPS}(\check{S}, \mathbb{Q})$ such that $\frac{d \mathbb{Q}}{d \underline{\mathbb{P}}} \in L^{\infty}$.

\subsection{Financial interpretation}

Before proving the main theorem, we give its financial interpretation. The measure EBAMM is an equivalent probability measure under which the conditional expectation of the bid price $\underline{S}_{t}^{i}$ given $\mathcal{F}_{t-1}$ is less than the ask price $\bar{S}_{t-1}^{i}$, and on the other hand the conditional expectation of the ask price $\bar{S}_{t}^{i}$ given $\mathcal{F}_{t-1}$ is more than the bid price $\underline{S}_{t-1}^{i}$. It can be seen as a generalization of the concept of an EMM in markets without friction. The no-arbitrage property is equivalent to the existence of an EBAMM. Nevertheless the desired equivalent condition would be the existence of a CPS. We obtain a counterpart of this condition, i.e. the existence of a supCPS and a subCPS under the same equivalent probability measure. The first one plays a role of a CPS when we have the short sale restriction, i.e. we can only have long positions in assets. The other one yields a CPS when we can only short sale assets. Notice that the bifurcation of CPS corresponds with the bifurcation of the price into bid and ask prices in our model.

In the proof of Theorem 2 the following results will be used. Their proofs can be found e.g. in Kabanov and Stricker (2001a).

Lemma 8 Let $X_{n}$ be a sequence of random vectors taking values in $\mathbb{R}^{d}$ such that $\lim \inf \left\|X_{n}(\omega)\right\|<\infty$ for almost all $\omega \in \Omega$. Then there is a sequence of random vectors $Y_{n}$ taking values in $\mathbb{R}^{d}$ satisfying the following conditions:

(1) $Y_{n}$ converges pointwise to $Y$ almost surely where $Y$ is a random vector taking values in $\mathbb{R}^{d}$,

(2) $Y_{n}(\omega)$ is a convergent subsequence of $X_{n}(\omega)$ for almost all $\omega \in \Omega$.

Proof See e.g. Kabanov and Stricker (2001a, Lemma 2) or Kabanov et al. (2002, Lemma 1).

Remark 8 The above claim can be formulated as follows: there exists an increasing sequence of integer-valued random variables $\sigma_{k}$ such that $X_{\sigma_{k}}$ converges a.s.

Lemma 9 (Kreps-Yan) Let $K \supseteq-L_{+}^{1}$ be a closed convex cone in $L^{1}$ such that $K \cap L_{+}^{1}=\{0\}$. Then there is a probability $\widetilde{P} \sim P$ with $\frac{d \widetilde{P}}{d P} \in L^{\infty}$ such that $E_{\tilde{P}} \xi \leq 0$ for all $\xi \in K$.

Proof See e.g. Kabanov and Stricker (2001a, Lemma 3) or Kabanov and Safarian (2009, Theorem 2.1.4). 
Proof of Theorem 2 In general, the proof adapts the approach in Kabanov and Stricker (2001a). However, there are significant differences. To show the closedness of $F_{t-1, t+k}$ we apply Lemma 8 but our reasoning on $\Omega_{2}$ differs from that in Kabanov and Stricker (2001a). Here we use the idea borrowed from Rygiel and Stettner (2012). In the next part, similarly as in Kabanov and Stricker (2001a), we apply Lemma 9. This allows us to obtain an EBAMM but only in the one-step model. To constract such a measure in a multi-period case we use induction on the length of the time interval. In the next implication we construct a counterpart of a CPS, i.e. we show that there exists a supCPS and a subCPS under the same equivalent probability measure. In order to obtain this condition we apply the theory of optimal stopping combined with the induction. Fortunately, by Theorem 1, this condition is sufficient for the absence of arbitrage. This is due to the fact that we split the strategy into two strategies, which consists of long and short positions only. Therefore we can use a supCPS and a subCPS to yield the no-arbitrage property.

(a) $\Rightarrow$ (b). By Lemma $1 \mathcal{A}_{t} \cap L_{+}^{0}\left(\mathcal{F}_{t}\right)=\{0\}$ for any $t=1, \ldots, T$ and using Lemma 6 also $F_{t} \cap L_{+}^{0}\left(\mathcal{F}_{t}\right)=\{0\}$ for any $t=1, \ldots, T$.

(b) $\Rightarrow$ (c). Trivial [Notice that also (a) $\Rightarrow$ (c) is obvious so we could skip condition (b), which we actually put here to make the analysis more transparent].

(c) $\Rightarrow(d)$. To prove this implication we will use a similar technique to Kabanov and Stricker (2001a) and especially Rygiel and Stettner (2012, Theorem 2.33). Take any $t, k$ such $1 \leq t \leq t+k \leq T$. We will show that the set $F_{t-1, t+k}$ is closed in the topology generated by convergence in probability $\mathbb{P}$. Take a sequence $\xi^{n} \in F_{t-1, t+k}$ such that $\xi^{n} \rightarrow \zeta$ in probability. It suffices to show that $\zeta \in F_{t-1, t+k}$. The sequence $\xi^{n}$ contains a subsequence convergent to $\zeta$ a.s. Thus, restricting to this subsequence we can assume that $\xi^{n} \rightarrow \zeta \mathbb{P}$-a.s. By Lemma 7 for any $n$ there exist $H_{t}^{n} \in L^{0}\left(\mathbb{R}^{d}, \mathcal{F}_{t-1}\right)$ and $r_{n} \in L_{+}^{0}\left(\mathcal{F}_{t+k}\right)$ such that

$\xi^{n}=-\left(H_{t}^{n}\right)^{+} \cdot \bar{S}_{t-1}+\left(H_{t}^{n}\right)^{-} \cdot \underline{S}_{t-1}+\left(H_{t}^{n}\right)^{+} \cdot \underline{S}_{t+k}-\left(H_{t}^{n}\right)^{-} \cdot \bar{S}_{t+k}-r_{n} \in F_{t-1, t+k}$,

which simply means that $x_{t-1, t+k}\left(H_{t}^{n}\right) \rightarrow \zeta \mathbb{P}$-a.s.

Consider first the situation on the set $\Omega_{1}:=\left\{\liminf \left\|H_{t}^{n}\right\|<\infty\right\} \in \mathcal{F}_{t-1}$. By Lemma 8 there exists an increasing sequence of integer-valued $\mathcal{F}_{t-1}$-measurable random variables $\tau_{n}$ such that $H_{t}^{\tau_{n}}$ is convergent a.s. on $\Omega_{1}$ and for almost all $\omega \in \Omega_{1}$ the sequence $H_{t}^{\tau_{n}(\omega)}(\omega)$ is a convergent subsequence of $H_{t}^{n}(\omega)$. Notice that $H_{t}^{\tau_{n}} \in$ $L^{0}\left(\mathbb{R}^{d}, \mathcal{F}_{t-1}\right)$ and $r_{\tau_{n}} \in L_{+}^{0}\left(\mathcal{F}_{t+k}\right)$. Let $\tilde{H}_{t}:=\lim _{n \rightarrow \infty} H_{t}^{\tau_{n}}$. Since $H_{t}^{\tau_{n}}$ is convergent, so are $\left(H_{t}^{\tau_{n}}\right)^{+}$and $\left(H_{t}^{\tau_{n}}\right)^{-}$. Moreover $\left(H_{t}^{\tau_{n}}\right)^{+} \rightarrow\left(\tilde{H}_{t}\right)^{+}$and $\left(H_{t}^{\tau_{n}}\right)^{-} \rightarrow\left(\tilde{H}_{t}\right)^{-}$. Hence also $r_{\tau_{n}}$ is convergent a.s. on $\Omega_{1}$. Define $\tilde{r}:=\lim _{n \rightarrow \infty} r_{\tau_{n}}$. Then

$$
\begin{aligned}
\zeta & =\lim _{n \rightarrow \infty}\left(-\left(H_{t}^{n}\right)^{+} \cdot \bar{S}_{t-1}+\left(H_{t}^{n}\right)^{-} \cdot \underline{S}_{t-1}+\left(H_{t}^{n}\right)^{+} \cdot \underline{S}_{t+k}-\left(H_{t}^{n}\right)^{-} \cdot \bar{S}_{t+k}-r_{n}\right) \\
& =\lim _{n \rightarrow \infty}\left(-\left(H_{t}^{\tau_{n}}\right)^{+} \cdot \bar{S}_{t-1}+\left(H_{t}^{\tau_{n}}\right)^{-} \cdot \underline{S}_{t-1}+\left(H_{t}^{\tau_{n}}\right)^{+} \cdot \underline{S}_{t+k}-\left(H_{t}^{\tau_{n}}\right)^{-} \cdot \bar{S}_{t+k}-r_{\tau_{n}}\right)
\end{aligned}
$$

and the above limit is equal to

$$
-\left(\tilde{H}_{t}\right)^{+} \cdot \bar{S}_{t-1}+\left(\tilde{H}_{t}\right)^{-} \cdot \underline{S}_{t-1}+\left(\tilde{H}_{t}\right)^{+} \cdot \underline{S}_{t+k}-\left(\tilde{H}_{t}\right)^{-} \cdot \bar{S}_{t+k}-\tilde{r} \in F_{t-1, t+k} .
$$


Consider now the situation on the set $\Omega_{2}:=\left\{\liminf \left\|H_{t}^{n}\right\|=\infty\right\} \in \mathcal{F}_{t-1}$. Define $G_{t}^{n}:=\frac{H_{t}^{n}}{\left\|H_{t}^{n}\right\|}, h_{n}:=\frac{r_{n}}{\left\|H_{t}^{n}\right\|}$ and notice that $G_{t}^{n} \in L^{0}\left(\mathbb{R}^{d}, \mathcal{F}_{t-1}\right)$ and $h_{n} \in L_{+}^{0}\left(\mathcal{F}_{t+k}\right)$. We get the convergence

$$
-\left(G_{t}^{n}\right)^{+} \cdot \bar{S}_{t-1}+\left(G_{t}^{n}\right)^{-} \cdot \underline{S}_{t-1}+\left(G_{t}^{n}\right)^{+} \cdot \underline{S}_{t+k}-\left(G_{t}^{n}\right)^{-} \cdot \bar{S}_{t+k}-h_{n} \rightarrow 0 .
$$

Just as on $\Omega_{1}$, by Lemma 8 there exists an increasing sequence of integer-valued $\mathcal{F}_{t-1}$-measurable random variables $\sigma_{n}$ such that $G_{t}^{\sigma_{n}}$ is convergent a.s. on $\Omega_{2}$ and for almost all $\omega \in \Omega_{2}$ the sequence $G_{t}^{\sigma_{n}(\omega)}(\omega)$ is a convergent subsequence of $G_{t}^{n}(\omega)$. Let $\tilde{G}_{t}:=\lim _{n \rightarrow \infty} G_{t}^{\sigma_{n}}$. As before, by the convergence of $G_{t}^{\sigma_{n}}$, also $\left(G_{t}^{\sigma_{n}}\right)^{+}$and $\left(G_{t}^{\sigma_{n}}\right)^{-}$ are convergent. Moreover $\left(G_{t}^{\sigma_{n}}\right)^{+} \rightarrow\left(\tilde{G}_{t}\right)^{+}$and $\left(G_{t}^{\sigma_{n}}\right)^{-} \rightarrow\left(\tilde{G}_{t}\right)^{-}$. Hence also $h_{\sigma_{n}}$ is convergent a.s. on $\Omega_{2}$. Define $\tilde{h}:=\lim _{n \rightarrow \infty} h_{\sigma_{n}}$. We get

$$
-\left(\tilde{G}_{t}\right)^{+} \cdot \bar{S}_{t-1}+\left(\tilde{G}_{t}\right)^{-} \cdot \underline{S}_{t-1}+\left(\tilde{G}_{t}\right)^{+} \cdot \underline{S}_{t+k}-\left(\tilde{G}_{t}\right)^{-} \cdot \bar{S}_{t+k}=\tilde{h} .
$$

Since $F_{t-1, t+k} \cap L_{+}^{0}\left(\mathcal{F}_{t+k}\right)=\{0\}$ we have $\tilde{h}=0 \mathbb{P}$-a.e. Therefore

$$
\left(\tilde{G}_{t}\right)^{+} \cdot\left(\underline{S}_{t+k}-\bar{S}_{t-1}\right)-\left(\tilde{G}_{t}\right)^{-} \cdot\left(\bar{S}_{t+k}-\underline{S}_{t-1}\right)=0 \quad \text { P-a.e. on } \Omega_{2} \text {. }
$$

Since $\tilde{G}_{t}(\omega) \neq 0$ a.e. on $\Omega_{2}$ [because $G_{t}^{\sigma_{n}}(\omega)$ is a convergent subsequence of $G_{t}^{n}(\omega)$ for almost all $\omega \in \Omega_{2}$, and $\left\|G_{t}^{n}(\omega)\right\|=1$ for almost all $\left.\omega \in \Omega_{2}\right]$, there exists a partition of $\Omega_{2}$ into at most $d$ disjoint subsets $\Omega_{2}^{i} \in \mathcal{F}_{t-1}$ such that $\tilde{G}_{t}^{i}(\omega) \neq 0$ a.s. on $\Omega_{2}^{i}$ (Such a partition can be achieved by choosing $\Omega_{2}^{1}:=\left\{\omega \in \Omega_{2}: \tilde{G}_{t}^{1}(\omega) \neq 0\right\}$, then continuing the partition on the set $\Omega_{2} \backslash \Omega_{2}^{1}$ by choosing $\Omega_{2}^{2}:=\left\{\omega \in \Omega_{2} \backslash \Omega_{2}^{1}: \tilde{G}_{t}^{2}(\omega) \neq 0\right\}$, and so on). Moreover, any non-empty set $\Omega_{2}^{i}$ can be divided into at most two disjoint subsets $\Omega_{2}^{i,+}:=\left\{\tilde{G}_{t}^{i}>0\right\}$ and $\Omega_{2}^{i,-}:=\left\{\tilde{G}_{t}^{i}<0\right\}$. For non-empty $\Omega_{2}^{i,+}$ and $\Omega_{2}^{i,-}$ define

$$
\begin{aligned}
\left(\bar{H}_{t}^{n}\right)^{p}:=\left(H_{t}^{n}\right)^{+}-\beta_{n}\left(\tilde{G}_{t}\right)^{+} \text {where } \beta_{n}:=\min _{j:\left(\tilde{G}_{t}^{j}\right)^{+}>0} \frac{\left(H_{t}^{n j}\right)^{+}}{\left(\tilde{G}_{t}^{j}\right)^{+}} \text {on } \Omega_{2}^{i,+}, \\
\left(\bar{H}_{t}^{n}\right)^{m}:=\left(H_{t}^{n}\right)^{-}-\beta_{n}\left(\tilde{G}_{t}\right)^{-} \text {where } \beta_{n}:=\min _{j:\left(\tilde{G}_{t}^{j}\right)^{-}>0} \frac{\left(H_{t}^{n j}\right)^{-}}{\left(\tilde{G}_{t}^{j}\right)^{-}} \text {on } \Omega_{2}^{i,-} .
\end{aligned}
$$

First we show that $\beta_{n}$ is a well-defined, non-negative, $\mathcal{F}_{t-1}$-measurable random variable. It suffices to consider the case of $\beta_{n}$ defined on $\Omega_{2}^{i,+}$. The other case is analogous. Notice that $\left(\tilde{G}_{t}^{i}\right)^{+} \neq 0$ on $\Omega_{2}^{i,+}$ but on some subsets of $\Omega_{2}^{i,+}$ there can exist another strictly positive coordinate of $\tilde{G}_{t}$. Hence we may divide $\Omega_{2}^{i,+}$ into disjoint subsets $\Omega_{2}^{i,+}\left(i_{1}, \ldots, i_{k}\right)$ where $1 \leq i_{1}<\cdots<i_{k} \leq d$ and $\tilde{G}_{t}^{j} \neq 0$ only for $j \in\left\{i_{1}, \ldots, i_{k}\right\}$. Notice that $\Omega_{2}^{i,+}\left(i_{1}, \ldots, i_{k}\right) \in \mathcal{F}_{t-1}$ and if $\Omega_{2}^{i,+}\left(i_{1}, \ldots, i_{k}\right) \neq \emptyset$ then on this sub$\operatorname{set} \beta_{n}=\max _{i_{1}, \ldots, i_{k}}\left\{\left(H_{t}^{n i_{1}}\right)^{+} /\left(\tilde{G}_{t}^{i_{1}}\right)^{+}, \ldots,\left(H_{t}^{n i_{k}}\right)^{+} /\left(\tilde{G}_{t}^{i_{k}}\right)^{+}\right\}$, which is a well-defined, $\mathcal{F}_{t-1}$-measurable random variable. Since $\Omega_{2}^{i,+}=\bigcup \Omega_{2}^{+}\left(i_{1}, \ldots, i_{k}\right)$ where the sum is 
finite, taken over all $1 \leq i_{1}<\cdots<i_{k} \leq d$ such that $\Omega_{2}^{+}\left(i_{1}, \ldots, i_{k}\right) \neq \emptyset$, it follows that $\beta_{n}$ is $\mathcal{F}_{t-1}$-measurable.

Finally, we set $\bar{H}_{t}^{n}:=\left(\bar{H}_{t}^{n}\right)^{p}-\left(\bar{H}_{t}^{n}\right)^{m}$ (equivalently for any $i=1, \ldots, d$ we could define $\bar{H}_{t}^{n i}=\left(\bar{H}_{t}^{n i}\right)^{p}$ on a non-empty $\Omega_{2}^{i,+}$ and $\bar{H}_{t}^{n i}=-\left(\bar{H}_{t}^{n i}\right)^{m}$ on a non-empty $\left.\Omega_{2}^{i,-}\right)$. Notice that $\left(\bar{H}_{t}^{n}\right)^{p} \geq 0$ and $\left(\bar{H}_{t}^{n}\right)^{m} \geq 0$. Indeed, consider the situation on any non-empty $\Omega_{2}^{i,+}$. For any $j=1, \ldots, d$ we have

$$
\left(H_{t}^{n j}\right)^{+}-\frac{\left(H_{t}^{n j}\right)^{+}}{\left(\tilde{G}_{t}^{j}\right)^{+}}\left(\tilde{G}_{t}^{j}\right)^{+}=0 \quad \text { and } \quad 0 \leq \beta_{n} \leq \frac{\left(H_{t}^{n j}\right)^{+}}{\left(\tilde{G}_{t}^{j}\right)^{+}}
$$

Hence $\left(\bar{H}_{t}^{n}\right)^{p} \geq 0$ and for almost all $\omega \in \Omega_{2}^{i,+}$ there exists at least one coordinate of $\left(\bar{H}_{t}^{n}\right)^{p}(\omega)$ which is now equal to zero. The situation on $\Omega_{2}^{i,-}$ is analogous. In fact, $\left(\bar{H}_{t}^{n}\right)^{+}=\left(\bar{H}_{t}^{n}\right)^{p}$ and $\left(\bar{H}_{t}^{n}\right)^{-}=\left(\bar{H}_{t}^{n}\right)^{m}$. Hence

$$
\begin{aligned}
x_{t-1, t+k}\left(\bar{H}_{t}^{n}\right)= & -\left(\bar{H}_{t}^{n}\right)^{+} \cdot \bar{S}_{t-1}+\left(\bar{H}_{t}^{n}\right)^{-} \cdot \underline{S}_{t-1}+\left(\bar{H}_{t}^{n}\right)^{+} \cdot \underline{S}_{t+k}-\left(\bar{H}_{t}^{n}\right)^{-} \cdot \bar{S}_{t+k} \\
= & -\left[\left(H_{t}^{n}\right)^{+}-\beta_{n}\left(\tilde{G}_{t}\right)^{+}\right] \cdot \bar{S}_{t-1}+\left[\left(H_{t}^{n}\right)^{-}-\beta_{n}\left(\tilde{G}_{t}\right)^{-}\right] \cdot \underline{S}_{t-1} \\
& +\left[\left(H_{t}^{n}\right)^{+}-\beta_{n}\left(\tilde{G}_{t}\right)^{+}\right] \cdot \underline{S}_{t+k}-\left[\left(H_{t}^{n}\right)^{-}-\beta_{n}\left(\tilde{G}_{t}\right)^{-}\right] \cdot \bar{S}_{t+k} \\
= & x_{t-1, t+k}\left(H_{t}^{n}\right) \\
& -\beta_{n}\left(-\tilde{G}_{t}^{+} \cdot \bar{S}_{t-1}+\tilde{G}_{t}^{-} \cdot \underline{S}_{t-1}+\tilde{G}_{t}^{+} \cdot \underline{S}_{t+k}-\tilde{G}_{t}^{-} \cdot \bar{S}_{t+k}\right) \\
= & x_{t-1, t+k}\left(H_{t}^{n}\right) .
\end{aligned}
$$

The last equality holds by (3). Hence $x_{t-1, t+k}\left(\bar{H}_{t}^{n}\right)=x_{t-1, t+k}\left(H_{t}^{n}\right) \mathbb{P}$-a.e. on $\Omega_{2}$ and for almost all $\omega \in \Omega_{2}$ at least one coordinate of $\bar{H}_{t}^{n}(\omega)$ is zero. However, this coordinate may be different for different $\omega \in \Omega_{2}$. Now we apply our procedure to the sequence $\bar{\xi}^{n}:=x_{t-1, t+k}\left(\bar{H}_{t}^{n}\right)-\bar{r}_{n} \rightarrow \zeta \mathbb{P}$-a.s. on $\Omega_{2}$. Our operations do not affect zero coordinates of the sequence $\bar{H}_{t}^{n}(\omega)$ for almost all $\omega \in \Omega_{2}$, so by iteration, after a finite number of steps, we construct the desired sequence.

(d) $\Rightarrow$ (e). Trivial.

(e) $\Rightarrow$ (f). To prove this implication we use some techniques from Rola (2013) combined with the construction of a measure by induction as in Rygiel and Stettner (2012, Corollary 2.35). Notice that for any random variable $\eta$ there exists a probability measure $P^{\prime} \sim \mathbb{P}$ such that $\frac{d P^{\prime}}{d \mathbb{P}} \in L^{\infty}$ and $\eta \in L^{1}\left(P^{\prime}\right)$. Property (d) is invariant under an equivalent change of probability. This allows us to assume without loss of generality that all $\underline{S}_{t}^{i}, \bar{S}_{t}^{i}$ are integrable. We will use induction on the length of the time interval. First we consider the time interval of the length 1. Fix any $t \in\{1, \ldots, T\}$ and define $\Psi_{t-1, t}:=\bar{F}_{t-1, t} \cap L^{1}\left(\mathcal{F}_{t}\right)$, a closed convex cone in $L^{1}\left(\mathcal{F}_{t}\right)$. Since $\Psi_{t-1, t} \cap L_{+}^{1}\left(\mathcal{F}_{t}\right)=\{0\}$, by Lemma 9 there exists a probability measure $\mathbb{Q}^{t} \sim \mathbb{P}$ on $\left(\Omega, \mathcal{F}_{t}\right)$ such that $\frac{d \mathbb{Q}^{t}}{d \mathbb{P}} \in L^{\infty}\left(\mathcal{F}_{t}\right)$ and $E_{\mathbb{Q}^{t}} \xi \leq 0$ for any $\xi \in \Psi_{t-1, t}$. In particular for 


$$
\begin{aligned}
& \xi_{t-1, t}^{i}=-H_{t}^{i} \bar{S}_{t-1}^{i}+H_{t}^{i} \underline{S}_{t}^{i}, \\
& \tilde{\xi}_{t-1, t}^{i}=H_{t}^{i} \underline{S}_{t-1}^{i}-H_{t}^{i} \bar{S}_{t}^{i},
\end{aligned}
$$

where $H_{t}=\left(0, \ldots, \mathbb{1}_{A}, \ldots, 0\right) \mathbb{P}$-a.e., we have $A \in \mathcal{F}_{t-1}$ and the value $\mathbb{1}_{A}$ is on the $i$ th position. For the case (4) this means that at time $t-1$, if the event $A$ holds we buy the $i$ th asset at price $\bar{S}_{t-1}^{i}$ and liquidate the portfolio at time $t$. For the case (5) the situation is opposite, i.e. first we short sale the $i$-th asset at time $t-1$ and then buy it at time $t$. Hence

$$
\begin{aligned}
& E_{\mathbb{Q}^{t}}\left[\left(\underline{S}_{t}^{i}-\bar{S}_{t-1}^{i}\right) \mathbb{1}_{A}\right] \leq 0, \\
& E_{\mathbb{Q}^{t}}\left[\left(\bar{S}_{t}^{i}-\underline{S}_{t-1}^{i}\right) \mathbb{1}_{A}\right] \geq 0 .
\end{aligned}
$$

Then $E_{\mathbb{Q}^{t}}\left(\underline{S}_{t}^{i} \mathbb{1}_{A}\right) \leq E_{\mathbb{Q}^{t}}\left(\bar{S}_{t-1}^{i} \mathbb{1}_{A}\right)$ and $E_{\mathbb{Q}^{t}}\left(\bar{S}_{t}^{i} \mathbb{1}_{A}\right) \geq E_{\mathbb{Q}^{t}}\left(\underline{S}_{t-1}^{i} \mathbb{1}_{A}\right)$ for any $i=$ $1, \ldots, d$ and $A \in \mathcal{F}_{t-1}$. Hence

$$
\begin{aligned}
& E_{\mathbb{Q}^{t}}\left(\underline{S}_{t}^{i} \mid \mathcal{F}_{t-1}\right) \leq E_{\mathbb{Q}^{t}}\left(\bar{S}_{t-1}^{i} \mid \mathcal{F}_{t-1}\right)=\bar{S}_{t-1}^{i}, \\
& E_{\mathbb{Q}^{t}}\left(\bar{S}_{t}^{i} \mid \mathcal{F}_{t-1}\right) \geq E_{\mathbb{Q}^{t}}\left(\underline{S}_{t-1}^{i} \mid \mathcal{F}_{t-1}\right)=\underline{S}_{t-1}^{i} .
\end{aligned}
$$

Obviously all $\underline{S}_{t}, \bar{S}_{t} \in L^{1}\left(\mathbb{Q}^{t}\right)$ since $\frac{d \mathbb{Q}^{t}}{d \mathbb{P}^{\mathbb{P}}}$ is bounded. In conclusion, there exists an EBAMM $\mathbb{Q}^{t}$ for the bid-ask process $(\underline{S}, \bar{S})$ where $\underline{S}=\left(\underline{S}_{j}\right)_{j=t-1}^{t}, \bar{S}=\left(\bar{S}_{j}\right)_{j=t-1}^{t}$ and $\frac{d \mathbb{Q}^{t}}{d \mathbb{P}} \in L^{\infty}$.

Assume now that the claim is true in a model with time interval $k \geq 1$. We will show that it is true in a model with time interval $k+1$. Fix any $t, k$ such that $1 \leq t \leq t+k \leq T$. We show that there exists an EBAMM in the market with the bid-ask process $(\underline{S}, \bar{S})$ where $\underline{S}=\left(\underline{S}_{j}\right)_{j=t-1}^{t+k}$ and $\bar{S}=\left(\bar{S}_{j}\right)_{j=t-1}^{t+k}$. By the induction hypothesis there exists an EBAMM $\mathbb{Q}^{t+k}$ in the market with the bid-ask process $\left(\left(\underline{S}_{j}\right)_{j=t}^{t+k},\left(\bar{S}_{j}\right)_{j=t}^{t+k}\right)$ such that $\frac{d \mathbb{Q}^{t+k}}{d \mathbb{P}} \in L^{\infty}$. Notice that condition (d) is invariant under an equivalent change of probability. Hence we can apply the same method as in the previous part to the probability space $\left(\Omega, \mathcal{F}_{t}, \mathbb{Q}_{\mid \mathcal{F}_{t}}^{t+k}\right)$ where $\mathbb{Q}_{\mid \mathcal{F}_{t}}^{t+k}$ denotes the measure $\mathbb{Q}^{t+k}$ restricted to $\mathcal{F}_{t}$. Then there exists a probability measure $\mathbb{Q}^{t} \sim \mathbb{Q}_{\mid \mathcal{F}_{t}}^{t+k}$ such that $\frac{d \mathbb{Q}^{t}}{d \mathbb{Q}_{\mid \mathcal{F}_{t}}^{t+k}} \in L^{\infty}$ and

$$
E_{\mathbb{Q}^{t}}\left(\underline{S}_{t}^{i} \mid \mathcal{F}_{t-1}\right) \leq \bar{S}_{t-1}^{i} \quad \text { and } \quad E_{\mathbb{Q}^{t}}\left(\bar{S}_{t}^{i} \mid \mathcal{F}_{t-1}\right) \geq \underline{S}_{t-1}^{i}
$$

for any $i=1, \ldots, d$. Define a probability measure $\mathbb{Q}$ on $\left(\Omega, \mathcal{F}_{t+k}\right)$ by

$$
\frac{d \mathbb{Q}}{d \mathbb{P}}:=\frac{d \mathbb{Q}^{t}}{d \mathbb{Q}_{\mid \mathcal{F}_{t}}^{t+k}} \frac{d \mathbb{Q}^{t+k}}{d \mathbb{P}}
$$

Notice that the density $\frac{d \mathbb{Q}^{t}}{d \mathbb{Q}_{\mid \mathcal{F}_{t}}^{t+k}}$ is bounded and $\mathcal{F}_{t}$-measurable, hence for any $j \in$ $\{t+1, \ldots, t+k\}$ and $i \in\{1, \ldots, d\}$ we have 


$$
\begin{aligned}
E_{\mathbb{Q}}\left(\underline{S}_{j}^{i} \mid \mathcal{F}_{j-1}\right) & =\frac{E_{\mathbb{P}}\left(\frac{d \mathbb{Q}^{t}}{d \mathbb{Q}_{\mid \mathcal{F}_{t}}^{t+k}} \frac{d \mathbb{Q}^{t+k}}{d \mathbb{P}} \underline{S}_{j}^{i} \mid \mathcal{F}_{j-1}\right)}{E_{\mathbb{P}}\left(\frac{d \mathbb{Q}^{t}}{d \mathbb{Q}_{\mid \mathcal{F}_{j-1}}^{t+k}} \frac{d \mathbb{Q}^{t+k}}{d \mathbb{P}} \mid \mathcal{F}_{j-1}\right)}=\frac{E_{\mathbb{P}}\left(\frac{d \mathbb{Q}^{t+k}}{d \mathbb{P}} \underline{S}_{j}^{i} \mid \mathcal{F}_{j-1}\right)}{E_{\mathbb{P}}\left(\frac{d \mathbb{Q}^{t+k}}{d \mathbb{P}} \mid \mathcal{F}_{j-1}\right)} \\
& =E_{\mathbb{Q}^{t+k}}\left(\underline{S}_{j}^{i} \mid \mathcal{F}_{j-1}\right) \leq \bar{S}_{j-1}^{i}
\end{aligned}
$$

and on the other hand

$$
\begin{aligned}
E_{\mathbb{Q}}\left(\bar{S}_{j}^{i} \mid \mathcal{F}_{j-1}\right) & =\frac{E_{\mathbb{P}}\left(\frac{d \mathbb{Q}^{t}}{d \mathbb{Q}_{\mid \mathcal{F}_{t}}^{t+k}} \frac{d \mathbb{Q}^{t+k}}{d \mathbb{P}} \bar{S}_{j}^{i} \mid \mathcal{F}_{j-1}\right)}{E_{\mathbb{P}}\left(\frac{d \mathbb{Q}^{t}}{d \mathbb{Q}_{\mid \mathcal{F}_{j-1}}^{t+k}} \frac{d \mathbb{Q}^{t+k}}{d \mathbb{P}} \mid \mathcal{F}_{j-1}\right)}=\frac{E_{\mathbb{P}}\left(\frac{d \mathbb{Q}^{t+k}}{d \mathbb{P}} \bar{S}_{j}^{i} \mid \mathcal{F}_{j-1}\right)}{E_{\mathbb{P}}\left(\frac{d \mathbb{Q}^{t+k}}{d \mathbb{P}} \mid \mathcal{F}_{j-1}\right)} \\
& =E_{\mathbb{Q}^{t+k}}\left(\bar{S}_{j}^{i} \mid \mathcal{F}_{j-1}\right) \geq \underline{S}_{j-1}^{i}
\end{aligned}
$$

Obviously all $\underline{S}_{t}, \bar{S}_{t} \in L^{1}(\mathbb{Q})$ since $\frac{d \mathbb{Q}}{d \mathbb{P}}$ is bounded. Moreover, $E_{\mathbb{Q}}\left(\bar{S}_{t}^{i} \mid \mathcal{F}_{t-1}\right)=$ $E_{\mathbb{Q}^{t}}\left(\bar{S}_{t}^{i} \mid \mathcal{F}_{t-1}\right)$ and $E_{\mathbb{Q}}\left(\underline{S}_{t}^{i} \mid \mathcal{F}_{t-1}\right)=E_{\mathbb{Q}^{t}}\left(\underline{S}_{t}^{i} \mid \mathcal{F}_{t-1}\right)$. By induction we conclude that there exists an EBAMM for the bid-ask process $\left(\left(\underline{S}_{t}\right)_{t=0}^{T},\left(\bar{S}_{t}\right)_{t=0}^{T}\right)$ such that $\frac{d \mathbb{Q}}{d \mathbb{P}} \in L^{\infty}$.

(f) $\Rightarrow(\mathrm{g})$. As in the previous implication, we use induction on the length of the time interval. First we consider the time interval of the length 1 . Fix any $t \in\{1, \ldots, T\}$ and define $\hat{S}=\left(\hat{S}_{j}\right)_{j=t-1}^{t}, \breve{S}=\left(\check{S}_{j}\right)_{j=t-1}^{t}$ by

$$
\begin{aligned}
& \hat{S}_{t}:=\underline{S}_{t}, \quad \hat{S}_{t-1}:=\max \left\{\underline{S}_{t-1}, E_{\mathbb{Q}^{t}}\left(\hat{S}_{t} \mid \mathcal{F}_{t-1}\right)\right\}, \\
& \check{S}_{t}:=\bar{S}_{t}, \quad \check{S}_{t-1}:=\min \left\{\bar{S}_{t-1}, E_{\mathbb{Q}^{t}}\left(\check{S}_{t} \mid \mathcal{F}_{t-1}\right)\right\}
\end{aligned}
$$

Notice that $\left(\hat{S}, \mathbb{Q}^{t}\right)$ is supCPS and $\left(\check{S}, \mathbb{Q}^{t}\right)$ is subCPS where $\mathbb{Q}^{t}$ is an EBAMM in the market with the bid-ask process $\left(\left(\underline{S}_{j}\right)_{j=t-1}^{t},\left(\bar{S}_{j}\right)_{j=t-1}^{t}\right)$ such that $\frac{d \mathbb{Q}}{d \mathbb{P}} \in L^{\infty}$.

Assume now that the claim is true in a model with time interval $k$ where $k \geq 1$. We will show that it is true in a model with time interval $k+1$. Fix any $t, k$ such that $1 \leq t \leq t+k \leq T$. We show that there exists a supCPS $(\hat{S}, \tilde{\mathbb{Q}})$ and a subCPS $(\breve{S}, \tilde{\mathbb{Q}})$ in the market with the bid-ask process $(\underline{S}, \bar{S})$ where $\underline{S}=\left(\underline{S}_{j}\right)_{j=t-1}^{t+k}$ and $\bar{S}=\left(\bar{S}_{j}\right)_{j=t-1}^{t+k}$. By the induction hypothesis there exists a supCPS $\left(\left(\hat{S}_{j}\right)_{j=t}^{t+k}, \mathbb{Q}^{t+k}\right)$ and a subCPS $\left(\left(\breve{S}_{j}\right)_{j=t}^{t+k}, \mathbb{Q}^{t+k}\right)$ in the market with the bid-ask process $\left(\left(\underline{S}_{j}\right)_{j=t}^{t+k},\left(\bar{S}_{j}\right)_{j=t}^{t+k}\right)$ such that $\frac{d \mathbb{Q}^{t+k}}{d \mathbb{P}} \in L^{\infty}$. Notice that condition (f) is invariant under an equivalent change of probability. Consider $\left(\Omega, \mathcal{F}_{t}, \mathbb{Q}_{\mid \mathcal{F}_{t}}^{t+k}\right)$ where $\mathbb{Q}_{\mid \mathcal{F}_{t}}^{t+k}$ denotes $\mathbb{Q}^{t+k}$ restricted to $\mathcal{F}_{t}$. Then by (f) we can assume that there exists an EBAMM $\mathbb{Q}^{t} \sim \mathbb{Q}_{\mid \mathcal{F}_{t}}^{t+k}$ such that $\frac{d \mathbb{Q}^{t}}{d \mathbb{Q}_{\mid \mathcal{F}_{t}}^{t+k}} \in L^{\infty}$. Define the processes $\hat{S}=\left(\hat{S}_{j}\right)_{j=t-1}^{t}, \check{S}=\left(\check{S}_{j}\right)_{j=t-1}^{t}$ as in (6), (7). Then $\left(\hat{S}, \mathbb{Q}^{t}\right),\left(\check{S}, \mathbb{Q}^{t}\right)$ are supCPS and subCPS respectively. Define the stopping time $\tau_{i}:=\min \left\{j \geq t-1 \mid \breve{S}_{j}^{i}=\check{S}_{t}^{i}\right\}$ for any $i \in\{1, \ldots, d\}$. Then by optimal stopping 
theory (see e.g. Björk 2009, Proposition 21.15) the process $\check{S}^{\tau}:=\left(\check{S}_{j \wedge \tau}\right)_{j=t-1}^{t}$ is a $\mathbb{Q}^{t}$-martingale where $\check{S}_{j \wedge \tau}:=\left(\check{S}_{j \wedge \tau_{1}}^{1}, \ldots, \check{S}_{j \wedge \tau_{d}}^{d}\right)$. It sufficies to show that $-\check{S}^{\tau}$ is a $\mathbb{Q}^{t}$-martingale. Notice that $-\check{S}_{t-1}^{i}=\max \left\{-\bar{S}_{t-1}^{i}, E_{\mathbb{Q}^{t}}\left(-\check{S}_{t}^{i} \mid \mathcal{F}_{t-1}\right)\right\}$. We now define a probability measure $\mathbb{Q}$ on $\left(\Omega, \mathcal{F}_{t+k}\right)$ by

$$
\frac{d \mathbb{Q}}{d \mathbb{P}}:=\frac{d \mathbb{Q}^{t}}{d \mathbb{Q}_{\mid \mathcal{F}_{t}}^{t+k}} \frac{d \mathbb{Q}^{t+k}}{d \mathbb{P}}
$$

The density $\frac{d \mathbb{Q}}{d \mathbb{P}}$ is bounded and $\frac{d \mathbb{Q}^{t}}{d \mathbb{Q}_{\mid \mathcal{F}_{t}}^{t+k}}$ is $\mathcal{F}_{t}$-measurable. Furthermore let $\hat{S}^{\prime}=$ $\left(\hat{S}_{j}^{\prime}\right)_{j=t-1}^{t+k}$ be of the form

$$
\hat{S}_{j}^{\prime}=\hat{S}_{j} \text { for any } j>t \text { and } \hat{S}_{j}^{\prime}=\check{S}_{j \wedge \tau} \text { for } j=t-1, t
$$

Notice that $\hat{S}_{t-1}^{\prime i}=\check{S}_{t-1 \wedge \tau_{i}}^{i}=\check{S}_{t-1}^{i}$ and $\hat{S}_{t}^{\prime i}=\check{S}_{t \wedge \tau_{i}}^{i}=\check{S}_{\tau_{i}}^{i}$. Hence the inequalities $\underline{S}_{j}^{i} \leq \hat{S}_{j}^{\prime i} \leq \bar{S}_{j}^{i}$ are also satisfied for $j=t-1, t$. Moreover, $\hat{S}^{\prime}=\left(\hat{S}_{j}^{\prime}\right)_{j=t-1}^{t+k}$ is a $\mathbb{Q}$-supermartingale. Indeed, by the definition of $\tau_{i}$ and taking into account (7), (8) we obtain in particular

$$
E_{\mathbb{Q}}\left(\hat{S}_{t+1}^{\prime i} \mid \mathcal{F}_{t}\right)=E_{\mathbb{Q}}\left(\hat{S}_{t+1}^{i} \mid \mathcal{F}_{t}\right)=E_{\mathbb{Q}^{t+k}}\left(\hat{S}_{t+1}^{i} \mid \mathcal{F}_{t}\right) \leq \hat{S}_{t}^{i} \leq \bar{S}_{t}^{i}=\check{S}_{t}^{i}=\check{S}_{\tau_{i}}^{i}=\hat{S}_{t}^{\prime i}
$$

for any $i=1, \ldots, d$. In an analogous way we can construct a $\mathbb{Q}$-submartingale. Define a stopping time $\sigma_{i}:=\min \left\{j \geq t-1 \mid \hat{S}_{j}^{i}=\hat{S}_{t}^{i}\right\}$. Then $\hat{S}^{\sigma}:=\left(\hat{S}_{j \wedge \sigma}\right)_{j=t-1}^{t}$ is a $\mathbb{Q}^{t}$-martingale where $\hat{S}_{j \wedge \sigma}:=\left(\hat{S}_{j \wedge \sigma_{1}}^{1}, \ldots, \hat{S}_{j \wedge \sigma_{d}}^{d}\right)$. Defining $\mathbb{Q}$ as in (8) and $\check{S}^{\prime}=\left(\check{S}_{j}^{\prime}\right)_{j=t-1}^{t+k}$ by

$$
\check{S}_{j}^{\prime}=\check{S}_{j} \quad \text { for any } j>t \text { and } \check{S}_{j}^{\prime}=\hat{S}_{j \wedge \tau} \text { for } j=t-1, t
$$

we get the desired $\mathbb{Q}$-submartingale. For more details about optimal stopping theory we refer e.g. to Chapter 21 in Björk (2009).

$(\mathrm{g}) \Rightarrow(\mathrm{a})$. This follows from Theorem 1 .

Remark 9 The conditions from Theorem 2 are also equivalent to another one: for any $1 \leq t \leq t+k \leq T$ there exists an EBAMM $\mathbb{Q}_{t-1}^{t+k}$ for the bid-ask process $\left\{\left(\underline{S}_{t-1}, \bar{S}_{t-1}\right),\left(\underline{S}_{t+k}, \bar{S}_{t+k}\right)\right\}$ such that $\frac{d \mathbb{Q}_{t-1}^{t+k}}{d \mathbb{P}} \in L^{\infty}$. Now for any $t, k$ with $1 \leq t \leq t+$ $k \leq T$ define $\mathcal{A}_{t-1, t+k}:=\mathcal{R}_{t-1, t+k}-L_{+}^{0}\left(\mathcal{F}_{t+k}\right)$ where $\mathcal{R}_{t-1, t+k}:=\left\{x_{t-1, t+k}\left(H_{t}\right) \mid\right.$ $\left.H_{t} \in L^{0}\left(\mathbb{R}^{d}, \mathcal{F}_{t-1}\right)\right\}$. Then under the assumption $\mathcal{A}_{t-1, t+k} \cap L_{+}^{0}\left(\mathcal{F}_{t+k}\right)=\{0\}$ the set $\mathcal{A}_{t-1, t+k}$ is closed in probability. It suffices to argue as in the proof of the implication (c) $\Rightarrow$ (d) of Theorem 2 . 
Remark 10 Assume that $T=1$ and let $\mathbb{Q}^{1}$ be an EBAMM. Define the Snell envelope $\hat{S}=\left(\hat{S}_{t}\right)_{t=0}^{1}$ of the bid process $\left(\underline{S}_{t}\right)_{t=0}^{1}$ as follows:

$$
\hat{S}_{1}:=\underline{S}_{1}, \quad \hat{S}_{0}:=\max \left\{\underline{S}_{0}, E_{\mathbb{Q}^{1}}\left(\hat{S}_{1} \mid \mathcal{F}_{0}\right)\right\} .
$$

Then $\left(\hat{S}, \mathbb{Q}^{1}\right)$ is a supCPS. Furthermore if we take the optimal stopping time

$$
\tau_{i}:=\min \left\{t \geq 0 \mid \underline{S}_{t}^{i}=\hat{S}_{1}^{i}\right\}
$$

then $\tilde{S}:=\left(\tilde{S}_{t}\right)_{t=0}^{1}$ is a $\mathbb{Q}^{1}$-martingale where $\tilde{S}_{t}^{i}:=\hat{S}_{t \wedge \tau_{i}}^{i}$ (see e.g. Björk 2009, Proposition 21.15). Moreover for any $i=1, \ldots, d$

$$
\tilde{S}_{0}^{i}=\hat{S}_{0}^{i}, \quad \tilde{S}_{1}^{i}=\hat{S}_{\tau_{i}}^{i}=\underline{S}_{1}^{i}
$$

hence $\underline{S}_{t}^{i} \leq \tilde{S}_{t}^{i} \leq \bar{S}_{t}^{i}$ and $\left(\tilde{S}, \mathbb{Q}^{1}\right)$ is a CPS.

These considerations allow us to formulate an easy consequence of Theorem 2 . The corollary below is also consistent with the results from the literature, e.g. in Cherny (2007, Example 2.9).

Corollary 1 If the time horizon $T$ is 1 then

$$
(N A) \Leftrightarrow(E B A M M) \Leftrightarrow(C P S) .
$$

Remark 11 Condition (g) of Theorem 2 says in particular that there exists a supCPS $(\hat{S}, \mathbb{Q})$. Define the Snell envelope $\tilde{S}$ of $\hat{S}$ as follows:

$$
\tilde{S}_{T}:=\hat{S}_{T}, \quad \tilde{S}_{t-1}:=\max \left\{\hat{S}_{t-1}, E_{\mathbb{Q}}\left(\tilde{S}_{t} \mid \mathcal{F}_{t-1}\right)\right\}
$$

for any $t=1, \ldots, T$. Notice that by optimal stopping theory, for any $i=1, \ldots, d$ $\tau_{i}:=\min \left\{t \geq 0 \mid \tilde{S}_{t}^{i}=\hat{S}_{t}^{i}\right\}$ is an optimal stopping time and $\tilde{S}^{\tau}:=\left(\tilde{S}_{t \wedge \tau}\right)_{t=0}^{T}$ is a $\mathbb{Q}$ martingale where $\tilde{S}_{t \wedge \tau}:=\left(\tilde{S}_{t \wedge \tau_{1}}^{1}, \ldots, \tilde{S}_{t \wedge \tau_{d}}^{d}\right)$ (see e.g. Björk 2009, Proposition 21.15). On the other hand, we cannot say that a pair $\left(\tilde{S}^{\tau}, \mathbb{Q}\right)$ is a CPS because we do not know whether $\underline{S}_{t}^{i} \leq \tilde{S}_{t \wedge \tau_{i}}^{i} \leq \bar{S}_{t}^{i}$ or not. We only know that $\underline{S}_{t \wedge \tau_{i}}^{i} \leq \tilde{S}_{t \wedge \tau_{i}}^{i} \leq \bar{S}_{t \wedge \tau_{i}}^{i}$.

Remark 12 In general the problem of equivalence between the existence of an EBAMM and a CPS in our model is open. It is not clear whether Corollary 1 can be extended to the case of any time horizon $T$. This problem also comes down to the following one. Assume that there exists a $\operatorname{supCPS}(\hat{S}, \mathbb{Q})$ and a $\operatorname{subCPS}(\check{S}, \mathbb{Q})$ under the same probability measure. Does there exist a CPS or not? Notice that the equivalence between the absence of arbitrage and the existence of a CPS for any $T$ in the case $d=1$ is a result of Grigoriev (2005, Corollary 2.9). Hence our question is relevant when $d>1$. Note that in general and in a more complex model we have the equivalence between the so-called robust no-arbitrage and the existence of a strictly CPS. This is the famous result of Schachermayer (2004). 
Remark 13 If we assume that there exists a strictly positive process $S=\left(S_{t}\right)_{t=0}^{T}$ such that $\bar{S}_{t}^{i}=\left(1+\lambda^{i}\right) S_{t}^{i}$ and $\underline{S}_{t}^{i}=\left(1-\mu^{i}\right) S_{t}^{i}$ where $0<\lambda^{i}, \mu^{i}<1$ then our model comes down to a stock market with proportional transaction costs. Indeed, the value process is then of the form

$$
\begin{aligned}
x_{t}(H)= & (H \cdot S)_{t}-\sum_{j=1}^{t} \lambda\left(\Delta H_{j}\right)^{+} S_{j-1}-\sum_{j=1}^{t} \mu\left(\Delta H_{j}\right)^{-} S_{j-1} \\
& -\lambda\left(H_{t}\right)^{-} S_{t}-\mu\left(H_{t}\right)^{+} S_{t} .
\end{aligned}
$$

\section{The Cox-Ross-Rubinstein model with bid-ask spreads}

The model introduced in Cox et al. (1979) is a very common example of a market without friction. Actually, it is the discrete version of the Black-Scholes model. In this section we introduce such a model in the case of markets with bid-ask spreads. Let $\left(\zeta_{t}\right)_{t=1}^{T}=\left(\underline{\zeta}_{t}, \bar{\zeta}_{t}\right)_{t=1}^{T}$ be a sequence of i.i.d. bivariate random variables defined on some probability space $(\Omega, \mathcal{F}, \mathbb{P})$ and concentrated at two points so that

$$
\mathbb{P}\left(\zeta_{t}=(\underline{u}, \bar{u})\right)=: p>0
$$

and

$$
\mathbb{P}\left(\zeta_{t}=(\underline{d}, \bar{d})\right)=1-p>0 .
$$

Let $\mathbb{F}=\left(\mathcal{F}_{t}\right)_{t=0}^{T}$ be a filtration such that $\mathcal{F}_{t}=\sigma\left(\zeta_{1}, \ldots, \zeta_{t}\right)$. Moreover, we set $\mathcal{F}_{0}=\{\emptyset, \Omega\}$ and $\mathcal{F}_{T}=\mathcal{F}$. It is convenient to assume that a money account $B_{t} \equiv 1$ for any $t=0, \ldots, T$. Define the dynamics of bid and ask scalar processes $\underline{S}, \bar{S}$ as follows:

$$
\underline{S}_{t}=\left(1+\underline{\zeta}_{t}\right) \bar{S}_{t-1} \text { and } \bar{S}_{t}=\left(1+\bar{\zeta}_{t}\right) \underline{S}_{t-1}
$$

For definiteness, we fix $\underline{d}<\underline{u}, \bar{d}<\bar{u}$. Moreover, assume that the bid and ask processes $\underline{S}, \bar{S}$ are strictly positive, i.e. $\underline{S}_{t}^{i}>0 \mathbb{P}$-a.e. for any $t=0, \ldots, T$. Hence we get $\underline{d}>-1$ and $\bar{d}>-1$. This corresponds e.g. to model of stock market. First, denote

$$
\begin{array}{ll}
\underline{S}_{t}^{u}:=\bar{S}_{t-1}(1+\underline{u}), & \bar{S}_{t}^{u}:=\underline{S}_{t-1}(1+\bar{u}), \\
\underline{S}_{t}^{d}:=\bar{S}_{t-1}(1+\underline{d}), & \bar{S}_{t}^{d}:=\underline{S}_{t-1}(1+\bar{d}) .
\end{array}
$$

This is illustrated in Fig. 1. Such a model can be seen as a generalization of the Cox-Ross-Rubinstein model to the case of bid-ask spreads. First we only consider the one-step model with the time moments $t-1$ and $t$. Notice that the general case is a concatenation of one-step models in a multiplicative way. Let us fix the initial bid-ask spread, i.e. $\left[\underline{S}_{t-1}, \bar{S}_{t-1}\right]$ where $t \in\{1, \ldots, T\}$. Our purpose is to estimate an EBAMM. Notice that in our model we should also know that $\underline{S}_{t} \leq \bar{S}_{t}$. In order to 


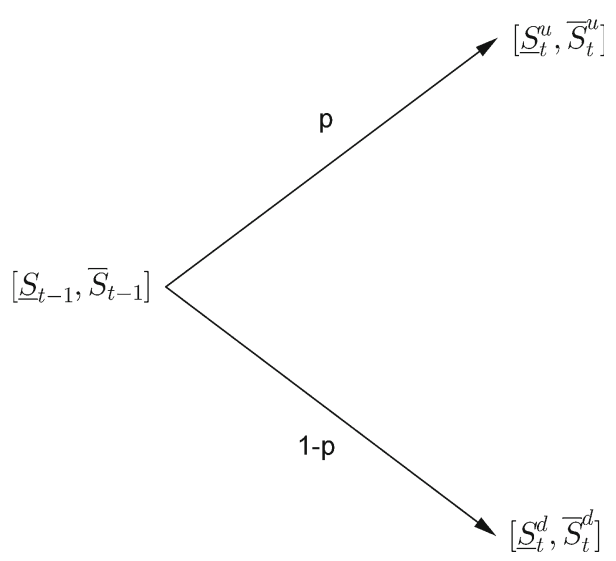

Fig. 1 The Cox-Ross-Rubinstein model with bid-ask spreads

ensure this we assume that $\frac{\bar{S}_{t-1}}{\underline{S}_{t-1}} \leq \frac{1+\bar{u}}{1+\underline{u}}$ and $\frac{\bar{S}_{t-1}}{\underline{S}_{t-1}} \leq \frac{1+\bar{d}}{1+\underline{d}}$. Denote the price spread at time $t$ by $\Delta_{t}:=\bar{S}_{t}-\underline{S}_{t}$. Then the following inequalities should be satisfied:

$$
1+\Delta_{t-1} \leq \frac{1+\bar{d}}{1+\underline{d}} \text { and } 1+\Delta_{t-1} \leq \frac{1+\bar{u}}{1+\underline{u}} .
$$

Denote by $\mathbb{P}^{*}$ an EBAMM. Let $p^{*}:=\mathbb{P}^{*}\left(\zeta_{t}=(\underline{u}, \bar{u}) \mid \mathcal{F}_{t-1}\right)=\mathbb{P}^{*}\left(\zeta_{t}=(\underline{u}, \bar{u})\right)$. Hence $\mathbb{P}^{*}\left(\zeta_{t}=(\underline{d}, \bar{d})\right)=1-p^{*}$. By definition $p^{*}$ should satisfy

$$
\begin{aligned}
& \bar{S}_{t-1}(1+\underline{u}) p^{*}+\bar{S}_{t-1}(1+\underline{d})\left(1-p^{*}\right) \leq \bar{S}_{t-1}, \\
& \underline{S}_{t-1}(1+\bar{u}) p^{*}+\underline{S}_{t-1}(1+\bar{d})\left(1-p^{*}\right) \geq \underline{S}_{t-1} .
\end{aligned}
$$

Hence we can estimate an EBAMM as follows:

$$
\frac{-\bar{d}}{\bar{u}-\bar{d}} \leq p^{*} \leq \frac{-\underline{d}}{\underline{u}-\underline{d}} .
$$

Notice that for the existence of an EBAMM we need to know that

$$
\frac{-\bar{d}}{\bar{u}-\overline{\bar{d}}} \leq \frac{-\underline{d}}{\underline{u}-\underline{d}} \quad \text { and } \quad 0<\frac{-\underline{d}}{\underline{u}-\underline{d}}, \quad \frac{-\bar{d}}{\bar{u}-\overline{\bar{d}}}<1 \text {. }
$$

These conditions ensure that there exists at least one $p^{*} \in(0,1)$. Then we get necessary and sufficient conditions for the existence of an EBAMM:

$$
\underline{d}<0<\bar{u} \text { and } \underline{d} \bar{u} \leq \bar{d} \underline{u} .
$$

By Theorem 2 these conditions are equivalent to the absence of arbitrage in the onestep Cox-Ross-Rubinstein model with bid-ask spreads. Now we are able to consider 


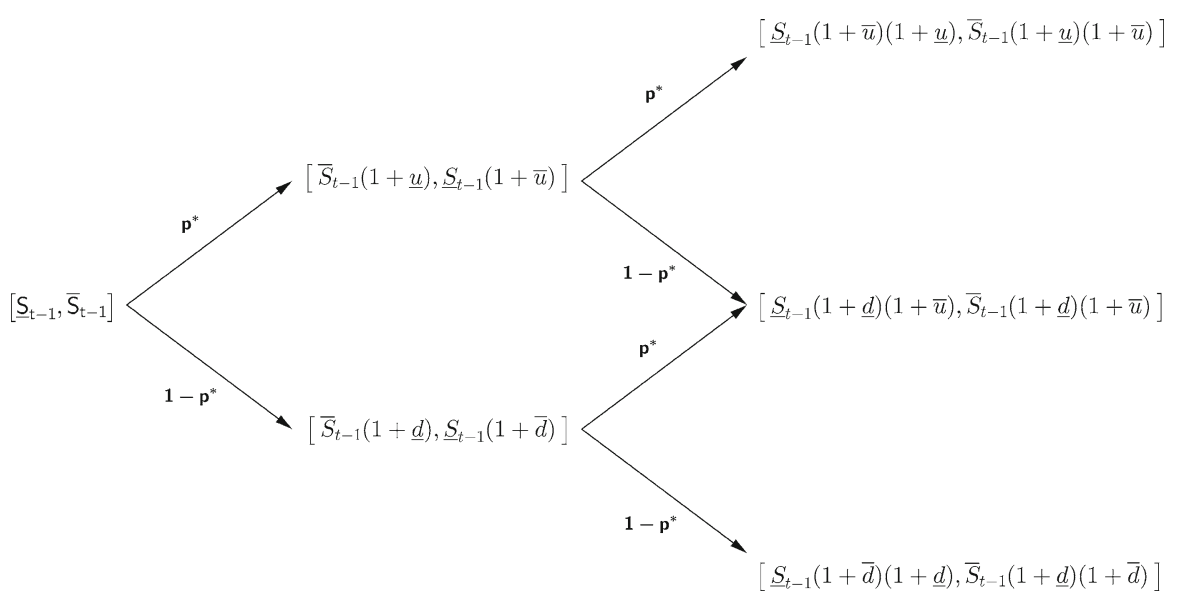

Fig. 2 The two-step CRR model with bid-ask spreads

a multi-period case with time horizon $T$. Firstly we should guarantee that the stock price interval follows a multiplicative binomial process. It also means that we want $\Omega$ to be a finite set of $2^{T}$ possible outcomes, all with positive probability. To guarantee this, it suffices to assume that

$$
\frac{1+\bar{u}}{1+\underline{u}}=\frac{1+\bar{d}}{1+\underline{d}} .
$$

Hence the inequalities (9) simplify to $1+\Delta \leq \frac{1+\bar{u}}{1+\underline{u}}=\frac{1+\bar{d}}{1+\underline{d}}$. Conditions (9) and (12) yield $\underline{S}_{t} \leq \bar{S}_{t}$ at any time $t$. An example of a two-step CRR model with bid-ask spreads and probabilities $p^{*}$ indicated is presented in Fig. 2.

Now we are in a position to formulate the main corollary of this chapter.

Corollary 2 Assume that in the CRR model with bid-ask spreads, $1+\Delta \leq \frac{1+\bar{u}}{1+\underline{u}}=\frac{1+\bar{d}}{1+\underline{d}}$ where $\Delta=\bar{S}_{0}-\underline{S}_{0}$. Then the inequalities in (11), i.e. $\underline{d}<0<\bar{u}$ and $\underline{d} \bar{u} \leq \bar{d} \underline{u}$ are necessary and sufficient for the existence of an EBAMM and actually for the absence of arbitrage. Moreover, $p^{*}$ is in $\left[\frac{-\bar{d}}{\bar{u}-\bar{d}}, \frac{-\underline{d}}{\underline{u}-\underline{d}}\right] \cap(0,1)$.

Finally, we illustrate our model by two simple examples.

Example 1 Consider the model with one risky asset and the time horizon $T=1$ presented in Fig. 3a. Notice that $\underline{d}=-\frac{3}{4}, \bar{d}=\underline{u}=0, \bar{u}=3$ and the conditions (11) are satisfied. Indeed, $\underline{d}=-\frac{3}{4}<0<3=\bar{u}$ and $\underline{d} \bar{u}=-\frac{9}{4} \leq 0=\bar{d} \underline{u}$. In this model there is no arbitrage and $p^{*} \in(0,1)$.

Example 2 Consider now the model with time horizon $T=1$ and one risky asset presented in Fig. 3b. In this model we have $\bar{u}=3, \bar{d}=-\frac{1}{4}, \underline{u}=\frac{1}{2}, \underline{d}=-\frac{3}{4}$ and by (10), $p^{*} \in\left[\frac{1}{13}, \frac{3}{5}\right]$. Notice that the conditions (11) are also satisfied. Indeed, $\underline{d}=-\frac{3}{4}<0<3=\bar{u}$ and $\underline{d} \bar{u}=-\frac{9}{4} \leq-\frac{1}{8}=\bar{d} \underline{u}$. 

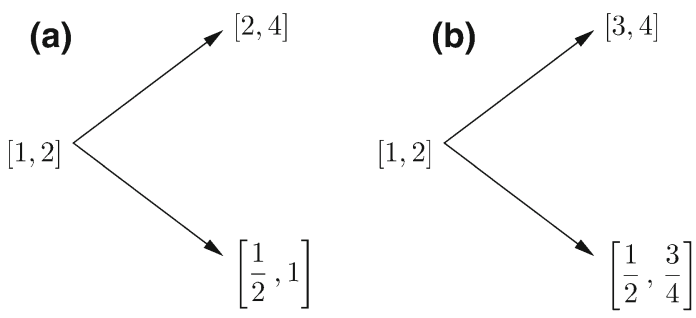

Fig. 3 Examples of one-step CRR models with bid-ask spreads

Acknowledgments The author would like to thank Professor Łukasz Stettner for numerous helpful suggestions and comments, and the anonymous referees for many useful and important remarks.

Open Access This article is distributed under the terms of the Creative Commons Attribution 4.0 International License (http://creativecommons.org/licenses/by/4.0/), which permits unrestricted use, distribution, and reproduction in any medium, provided you give appropriate credit to the original author(s) and the source, provide a link to the Creative Commons license, and indicate if changes were made.

\section{References}

Björk, T.: Arbitrage Theory in Continuous Time, 3rd edn. Oxford: Oxford University Press (2009)

Cherny, A.: General arbitrage pricing model: II—transaction costs. In: Séminaire de Probabilités XL. Lectures Notes in Mathematics, vol. 1899, pp. 447-461. Berlin: Springer (2007)

Cox, J.C., Ross, S.A., Rubinstein, M.: Option pricing: a simplified approach. J Financ Econ 7, 229-263 (1979)

Delbaen, F., Schachermayer, W.: The Mathematics of Arbitrage. Berlin: Springer (2006)

Dolinsky, Y., Soner, H.M.: Robust hedging with proportional transaction costs. Finance Stoch 18, 327-347 (2014)

Grigoriev, P.G.: On low dimensional case in the fundamental asset pricing theorem with transaction costs. Stat Decis 23, 33-48 (2005)

Jouini, E., Kallal, H.: Martingales and arbitrage in securities markets with transaction costs. J Econ Theory 66, 178-197 (1995)

Kabanov, Y.M., Rásonyi, M., Stricker, C.: No-arbitrage criteria for financial markets with efficient friction. Finance Stoch 6, 371-382 (2002)

Kabanov, Y.M., Safarian, M.: Markets with Transaction Costs. Mathematical Theory. Berlin: Springer (2009)

Kabanov, Y.M., Stricker, C.: A teacher's note on no-arbitrage criteria. In: Séminaire de Probabilités XXXV. Lecture Notes in Mathematics, vol. 1755, pp. 149-152 (2001)

Kabanov, Y.M., Stricker, C.: The Harrison-Pliska arbitrage pricing theorem under transaction costs. J Math Econ 35, 185-196 (2001)

Rola, P.: Arbitrage in markets without shortselling with proportional transaction costs. Appl Math (Warsaw) 40, 281-295 (2013)

Rygiel, A., Stettner, Ł.: Arbitrage for simple strategies. Appl Math (Warsaw) 39, 379-412 (2012)

Schachermayer, W.: The fundamental theorem of asset pricing under proportional transaction costs in finite discrete time. Math Finance 14, 19-48 (2004) 